\title{
Species and thermal radiation on micropolar hydromagnetic dusty fluid flow across a paraboloid revolution
}

\author{
M. Gnaneswara Reddy ${ }^{1} \cdot$ M. Ferdows ${ }^{2}$
}

Received: 17 July 2019 / Accepted: 1 January 2020 / Published online: 31 January 2020

(C) The Author(s) 2020

\begin{abstract}
Hydromagnetic flow of energy and species transfer behaviour of micropolar dusty fluid across a paraboloid revolution has been investigated. Heat and mass transfer phenomena are inspected through radiation, joule heating and chemical reaction. The boundary layer equations are modelled and transformed to a system of ODE'S with the aid of similarity transformations and solved numerically by utilizing Runge-Kutta integration scheme. The graphical analysis has been emphasized for the fluid and dust phase velocity, angular velocity, energy and species fields to the influence of sundry dynamical flow quantities. In addition, friction factor, Nusselt number and Sherwood number are presented in plots and tables and discussed elaborately and nice validation is emphasized. The obtained numerical results are checked with the earlier published articles. The boundary layers of angular velocity, temperature and concentration distributions increase for escalating values of magnetic parameter. For escalating values of material parameter, velocity profile increases for both momentum velocity and dusty velocity and opposite trend is seen in angular velocity profile. Concentration and boundary layer thickness diminish for escalating values of kr. Local friction factor declines for boost up values of magnetic parameter and porosity permeability parameter. The rate of heat transfer decreases for increasing values of Eckert number and radiation parameter, and rate of mass transfer increases for increasing values of Schmidt number and chemical reaction parameter.
\end{abstract}

Keywords Magnetic field $\cdot$ Porosity $\cdot$ Radiation $\cdot$ Micropolar dusty fluid $\cdot$ Heat transfer $\cdot$ Paraboloid revolution

\section{List of symbols}

A Non-negative number

$B_{0} \quad$ Magnetic field strength

$\mathrm{Bi}$ Biot number

C Concentrations of fluid

$C_{\mathrm{f}} \quad$ Friction factor

$C_{\mathrm{p}} \quad$ Concentrations of dust particles

$C_{\mathrm{mf}} \quad$ Specific heat of dust particles

$C_{\infty} \quad$ Ambient fluid concentration

$D_{\mathrm{m}} \quad$ Mass diffusivity coefficient

Ec Eckert number

$F \quad$ Dusty fluid

\section{Ferdows}

ferdowsmohammad@yahoo.com

M. Gnaneswara Reddy

mgrmaths@gmail.com

1 Department of Mathematics, Acharya Nagarjuna University Ongole Campus, Ongole 523 001, India

2 Research Group of Fluid Flow Modelling and Simulation, Department of Applied Mathematics, University of Dhaka, Dhaka 1000, Bangladesh
$\mathrm{Gr}_{\mathrm{b}} \quad$ Thermal buoyancy parameter

$\mathrm{Gr}_{\mathrm{c}} \quad$ Solutal buoyancy parameter

$\mathrm{Kr}^{*}$ Chemical reaction coefficient

$M \quad$ Magnetic parameter

$N \quad$ Microrotation velocity

$\mathrm{Nr}$ Radiation parameter

$N_{\text {ux }} \quad$ Nusselt number

Pr Prandtl number

$R \quad$ Material parameter

$\mathrm{Re}_{\mathrm{x}}$ Reynolds number

Sc Schmidt number

$\mathrm{Sh}_{\mathrm{x}} \quad$ Sheer wood number

$T$ Temperature of fluid

$T_{\mathrm{f}} \quad$ Convective fluid Temperature

$T_{\mathrm{p}} \quad$ Temperature of dust particles

$T_{\infty} \quad$ Ambient fluid temperature

$a \quad$ Stretching rate

$b \quad$ Non-negative number

$f \quad$ Fluid

$g$ Gravitational acceleration

$h_{\mathrm{f}} \quad$ Convective heat transfer

$k \quad$ Vertex viscosity 
$k_{1} \quad$ Stokes drag constant

$k_{\mathrm{f}} \quad$ Thermal conductivity of the fluid

$k_{\mathrm{p}} \quad$ Porous permeability parameter

$\mathrm{kr} \quad$ Chemical reaction parameter

j Microinertia per unit mass

$l \quad$ Mass concentration of dust particle

$m \quad$ Velocity power law index

$m_{0} \quad$ Boundary parameter

$q_{\mathrm{r}} \quad$ Radiative heat flux

$r \quad$ Mass of dust particle

$s \quad$ Number density of dust particles

$u \quad$ Velocity components of liquid fluid along $x$ axis

$u_{\mathrm{p}} \quad$ Velocity components of dusty fluid along $x$ axis

$u_{\mathrm{w}} \quad$ Stretching velocity

$v \quad$ Velocity components of fluid along $y$ axis

$v_{\mathrm{p}} \quad$ Velocity components of dusty fluid along $y$ axis

$\alpha \quad$ Upper horizontal sphere of paraboloid revolution

$\beta \quad$ Thermal expansion coefficient

$\beta^{*} \quad$ Solutal expansion coefficient

$\beta_{\mathrm{v}} \quad$ Fluid particle interaction parameter for velocity

$\beta_{\mathrm{t}} \quad$ Fluid particle interaction parameter for temperature

$\beta_{\mathrm{c}} \quad$ Fluid particle interaction parameter for concentration

$\gamma \quad$ Specific heat coefficient

$\gamma^{*} \quad$ Spin gradient viscosity

$\sigma \quad$ Electrical conductivity

$\rho \quad$ Effective density of fluid

$\rho_{\mathrm{p}} \quad$ Density of dust particles

$\mu \quad$ Dynamic viscosity of fluid

$v \quad$ Kinematic viscosity of fluid

$\tau_{\mathrm{c}} \quad$ Concentration equilibrium time

$\tau_{\mathrm{t}} \quad$ Thermal equilibrium time

$\tau_{\mathrm{v}} \quad$ Relaxation time of dust particle for velocity

$\xi \quad$ Stream function for fluid phase velocity

$\xi_{\mathrm{p}} \quad$ Stream function for dust phase velocity

$\theta \quad$ Dimension less temperature for fluid phase

$\theta_{\mathrm{p}} \quad$ Dimension less temperature dust phase

$\phi \quad$ Dimension less concentration for fluid phase

$\phi_{\mathrm{p}}$ Dimension less concentration dust phase

\section{Introduction}

Non-Newtonian fluids have been one of the most challenging and interesting areas in research for engineers, physicists, mathematicians and numerical simulates. Navier-Stokes theory cannot illuminate effectively the flow premises of polyamide fluids, emulsion suspensions, and liquids comprise definite additives. Out of these, the micropolar fluid is first presented in Eringen [1]. Micropolar fluid is a fluid which consists of microstructure applicable to a group of fluids along unsymmetrical stress tensor. A review of the subject and applications of micropolar fluid mechanics was mentioned by Peddison and McNitt [2], Khonsari and Brewe [3], Qukaszewicz [4]. Das [5] invoked the impact of hydromagnetic micropolar fluid across a rotating frame. Dulal pal and Sewli Chatterjee [6] studied the 2D flow of a micropolar fluid through a permeable stretching sheet, and the equations are employed numerically with finite difference method using quasi-linearization technique. Asma Khalid et al. [7] studied hydromagnetic micropolar couple stress fluid in a porous medium. By obtaining solutions, they are using perturbation technique. Hayat et al. [8] scrutinized steady 2D micropolar nanofluid across a stretching sheet. Gnaneswara Reddy and Rama Subba Reddy [9] studied heat transfer effects on MHD micropolar fluid over a vertical stretching surface. An unsteady two-dimensional MHD micropolar fluid flow over vertical plate analysed Gnaneswara Reddy [10]. They are resolved analytically by using perturbation method.

Alloying of micrometre-sized particles (dust particles) in the base fluids is also used to improve the thermal conductivity of the base liquid which is called dusty fluid. It has several applications in the field of combustion, purification of crude oil, electrostatic precipitation and fluid droplet sprays, etc. Saffman [11] has described the heat transfer effects on particle suspension flow. Chamkha [12] investigated the unsteady two-dimensional magnetohydrodynamic dusty flow across a circular pipe. Influence of heat transfer effects on Marangoni convective Dusty Casson fluid was scrutinized by Mahanthesh and Gireesha [13]. Mamata et al. [14] analysed magnetohydrodynamic flow of Fourier heat flux over cylinder. They are plotting graphical representations for the combination of nanoparticles Water+Silver and Water+ Graphene. Scrutinization of micropolar dusty nanofluid across a stretching sheet addressed Ghadikolaei et al. [15]. Gnaneswara Reddy et al. [16] invoked the phenomena of heat transfer impacts on radiative hydromagnetic Oldroyd-B dusty fluid across two different geometries. Crossdiffusion effects on MHD nonlinear radiation in two nonNewtonian fluids have been examined by Gnaneswara Reddy et al. [17]. Basma et al. [18] studied the slip flow of titanium and ferromagnetic nanoparticles along with dusty fluid.

Combined heat and mass transfer phenomena are found everywhere in nature, and it is important in all the branches of science and technology. In many practical applications, there exist significant temperature and concentration variations between the surface of the hot body and the free stream. It is a major area of interest, and it has assumed practical importance in engineering devices like heat exchangers, solar collectors, nuclear reactors and electronic equipments. Heat transfer effects on magnetohydrodynamic nanofluid were analysed by Sajid et al. [19]. They are obtaining solution by using homotopy analytical method. Dat et al. [20] examined the impact of $\mathrm{Al}_{2} \mathrm{O}_{3}$ nanoparticles with various shapes on thermal characteristics of nanofluid within a 
permeable space. Plane Poiseuille nanofluid with heat and mass transfer in a channel is examined by Alamri et al. [21]. Bhatti et al. [22] have been examined effects of heat transfer on particle-fluid suspension induced by metachronal wave. Bhatti et al. [23] scrutinized coagulation and endoscopy analysis for a particulate fluid suspension. Ellahi et al. [24] investigated heat transfer impacts on Electro-Osmotic Couette-Poiseuille Flow of MHD Power Law Nanofluid with Entropy Generation. Khan et al. [25] deliberated by heat generation in peristalsis flow of MHD nanofluids filled in an asymmetric channel is proposed. Sheikholeslami et al. [26] analyse the thermal behaviour of alumina nanofluid in a duct. Raei [27] explored the stabilized $\gamma-\mathrm{Al}_{2} \mathrm{O}_{3} /$ water nanofluids have been examined at the concentrations of 0.05 and $0.15 \mathrm{vol} \%$ with variation of flow rates. Monge-Palacios et al. [28] presented that cluster representation for fluids is an effective method to gain more fundamental insight into the nature of heat transfer in complex chemical systems such as nanofluids. Sheikholeslami et al. [29] have reported $\mathrm{Fe}_{3} \mathrm{O}_{4}$-water nanofluid flow in a cavity with constant heat flux is investigated 10 using a control volume-based finite element method. Sheikholeslami et al. [30] explored the heat transfer effects on magnetohydrodynamic nanoparticle through exergy analysis. Rafatijo et al. [31] developed a relationship for the ratio of the frequencies of termolecular to bimolecular collisions in terms of the temperature, density, and collision times. Sheikholeslami [32] explored the effect of magnetic field in a porous thermal energy storage enclosure using $\mathrm{CuO}$ nanoparticles. Galerkin finite element method is employed for this unsteady problem.

The study of boundary layer fluid flow has broad range of applications in science, industry and aviation. Published information on horizontal stretching sheet, circular cylinder, and two different geometries has been reported in [33-36]. Mather [37] was first investigating the moment of viscous fluid across a paraboloid. There are so many practical applications in paraboloid revolution, mainly used in automobile headlights, solar furnaces, radar, and radio relay stations heater, satellite dishes, automobile headlights, McDonald's Arches Fountains path of a Bal. Lee [38] presented a boundary layer flow of thin needle over a paraboloid. Twodimensional Blasius flow of Carreau flow on upper horizontal sphere of paraboloid revolution has been deliberated by Animasun and Pop [39]. They are obtaining numerically by using bvp5c MATLAB package. Kumaran et al. [40] developed a buoyancy model for two non-Newtonian fluids across a revolution of paraboloid. The researchers were plotted graphical representations for both the non-Newtonian (Casson and Carreau) fluids. Khan et al. [41] described biconvection flow of Carreau nanofluid across an upper horizontal sphere of paraboloid revolution. It is concluded that for escalating values of $\mathrm{Ha}$ and $\mathrm{Rb}$, there is an enhancement in the friction factor.
Many investigators concentrate all the above researches on analysing heat and mass transfer nature of flows by taking some physical effects with the normal substantial models. But in the present model, we study the flow of a micropolar dusty fluid across a paraboloid. Here we built up the influence of thermal radiation, chemical reaction, magnetic field, porosity parameter, joule heating. The boundary layer governing differential equations are modelled and transformed to a system of nonlinear ODE'S with the aid of similarity transformations. The final controlled equations along boundary restrictions are resolved numerically by Runge-Kutta method. The graphical results are elaborated for the fluid and dust phase velocity, angular velocity, temperature and concentration fields through the plots of non-dimensional quantities for sundry values of arising flow parameters. In addition, the physical quantities of interest namely friction factor, Nusselt and Sherwood numbers are presented in plots and tables and discussed elaborately to the impacts of multiple flow parameters.

\section{Modelling}

Consider a steady magnetohydrodynamic 2D flow of micropolar dusty fluid past a non-melting upper part of the revolving paraboloid. The flow is confined over the region $A(x+b)^{\frac{1-\mathrm{m}}{2}} \leq y<\infty$, and the revolving paraboloid is stretched with the velocity $u_{\mathrm{w}}=a(x+b)^{\mathrm{m}}$; hence, starting point of the flow at the slot is a function $y=A(x+b))^{1-\mathrm{m}_{/ 2}}$.

Here horizontal surface is located along $x$-direction and $y$-axis is normal to it. A uniform magnetic field of strength $B_{0}$ is applied in the flow direction as displayed in Fig. 1. Magnetic Reynolds number as low as conceivable to disregard the induce magnetic field, radiation, joule heating along with

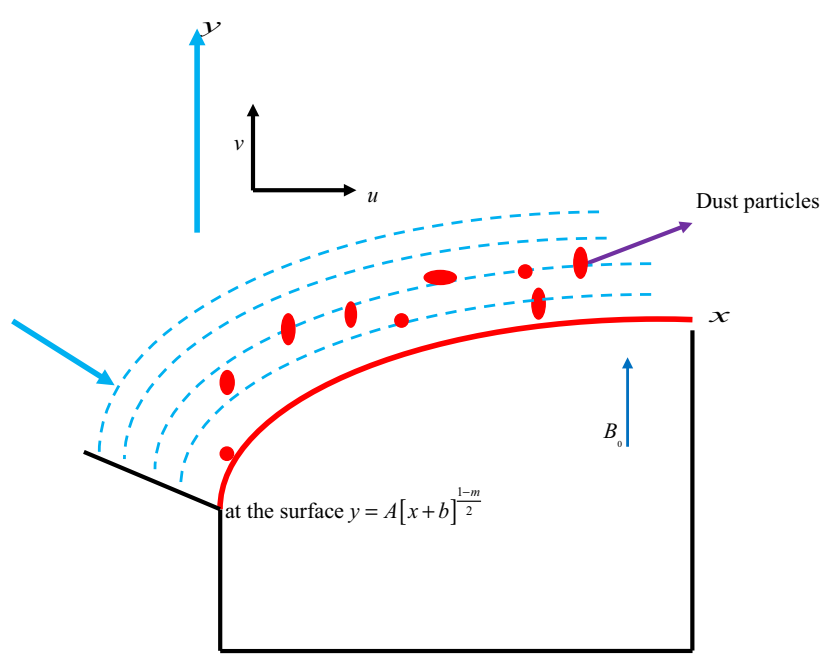

Fig. 1 Geometry of the problem 
chemical reaction effects are taken into account; under these assumptions, the governing equations of the flow are

The governing equations with stream function $\xi$ are as follows [40-42]:

$$
\begin{aligned}
& \frac{\partial^{2} \xi}{\partial x \partial y}-\frac{\partial^{2} \xi}{\partial y \partial x}=0 \\
& \frac{\partial \xi}{\partial y} \frac{\partial^{2} \xi}{\partial x \partial y}-\frac{\partial \xi}{\partial x} \frac{\partial^{2} \xi}{\partial y^{2}}-\left[\frac{\partial}{\partial x}\left(g \beta x\left(\frac{m+1}{2}\right)\left(T-T_{\infty}\right)\right)\right. \\
&\left.\quad+\frac{\partial}{\partial x}\left(g \beta^{*} x\left(\frac{m+1}{2}\right)\left(C-C_{\infty}\right)\right)\right] \\
&=\left(v+\frac{k}{\rho}\right) \frac{\partial^{3} \xi}{\partial y^{3}}-\frac{\sigma B_{0}^{2}}{\rho} \frac{\partial \xi}{\partial y}+\frac{k_{1} s}{\rho}\left(\frac{\partial \xi_{\mathrm{p}}}{\partial y}-\frac{\partial \xi}{\partial y}\right) \\
& \quad+\frac{k}{\rho} \frac{\partial N}{\partial y}-\frac{v}{k_{\rho}} \frac{\partial \xi}{\partial y}
\end{aligned}
$$

$\frac{\partial \xi}{\partial y} \frac{\partial N}{\partial x}-\frac{\partial \xi}{\partial x} \frac{\partial N}{\partial y}=\frac{\gamma^{*}}{\rho j} \frac{\partial^{2} N}{\partial y^{2}}-\frac{k}{\rho j}\left(2 N+\frac{\partial^{2} \xi}{\partial y^{2}}\right)$,

$\frac{\partial \xi}{\partial y} \frac{\partial T}{\partial x}-\frac{\partial \xi}{\partial x} \frac{\partial T}{\partial y}=\frac{k_{\mathrm{f}}}{\rho c_{\mathrm{p}}} \frac{\partial^{2} T}{\partial y^{2}}-\frac{1}{\rho c_{\mathrm{p}}} \frac{\partial q_{\mathrm{r}}}{\partial y}+\frac{1}{c_{\mathrm{p}}}\left(v+\frac{k}{\rho}\right)\left(\frac{\partial^{2} \xi}{\partial y^{2}}\right)^{2}$

$$
+\frac{\sigma B_{0}^{2}(x)}{\rho c_{\mathrm{p}}}\left(\frac{\partial \xi}{\partial y}\right)^{2}+\frac{\rho_{\mathrm{p}} C_{\mathrm{m}}}{\tau_{\mathrm{t}}}\left(T_{\mathrm{p}}-T\right)+\frac{\rho_{\mathrm{p}}}{\tau_{\mathrm{v}}}\left(\frac{\partial \xi_{\mathrm{p}}}{\partial y}-\frac{\partial \xi}{\partial y}\right)^{2}
$$

$\rho c_{\mathrm{p}}\left(\frac{\partial \xi}{\partial y} \frac{\partial C}{\partial x}-\frac{\partial \xi}{\partial x} \frac{\partial C}{\partial y}\right)=D_{\mathrm{m}} \frac{\partial^{2} C}{\partial y^{2}}-\mathrm{Kr}^{*}\left(C-C_{\infty}\right)+\frac{\rho_{\mathrm{p}} c_{\mathrm{p}}}{\tau_{\mathrm{c}}}\left(C_{\mathrm{p}}-C\right)$

$\frac{\partial^{2} \xi_{\mathrm{p}}}{\partial x \partial y}-\frac{\partial^{2} \xi_{\mathrm{p}}}{\partial y \partial x}=0$

$\rho_{\mathrm{p}}\left(\frac{\partial \xi_{\mathrm{p}}}{\partial y} \frac{\partial^{2} \xi_{\mathrm{p}}}{\partial x \partial y}-\frac{\partial \xi_{\mathrm{p}}}{\partial x} \frac{\partial^{2} \xi_{\mathrm{p}}}{\partial y^{2}}\right)=k_{1} s\left(\frac{\partial \xi}{\partial y}-\frac{\partial \xi_{\mathrm{p}}}{\partial y}\right)$

$\rho_{\mathrm{p}} c_{\mathrm{m}}\left(\frac{\partial \xi_{\mathrm{p}}}{\partial y} \frac{\partial T_{\mathrm{p}}}{\partial x}-\frac{\partial \xi_{\mathrm{p}}}{\partial x} \frac{\partial T_{\mathrm{p}}}{\partial y}\right)=-\frac{\rho_{\mathrm{p}} c_{\mathrm{pf}}}{\tau_{\mathrm{t}}}\left(T_{\mathrm{P}}-T\right)$

$\rho_{\mathrm{p}} c_{\mathrm{m}}\left(\frac{\partial \xi_{\mathrm{p}}}{\partial y} \frac{\partial C_{\mathrm{p}}}{\partial x}-\frac{\partial \xi_{\mathrm{p}}}{\partial x} \frac{\partial C_{\mathrm{p}}}{\partial y}\right)=-\frac{\rho_{\mathrm{p}} c_{\mathrm{pf}}}{\tau_{\mathrm{c}}}\left(C_{\mathrm{p}}-C\right)$

along the boundary conditions $u=u_{\mathrm{w}}=a(x+b)^{\mathrm{m}}, v=0$,

$N=-m_{0} \frac{\partial u}{\partial y},-k_{\mathrm{f}} \frac{\partial T}{\partial y}=h_{\mathrm{f}}\left(T_{\mathrm{f}}-T\right)$,

$C=C_{\mathrm{w}}$ at $y=A(x+b)^{\frac{1-\mathrm{m}}{2}}$

$u \rightarrow 0, N \rightarrow 0, u_{\mathrm{p}} \rightarrow 0, v_{\mathrm{p}} \rightarrow v$,

$T \rightarrow T_{\infty}, T_{\mathrm{p}} \rightarrow T_{\infty}, C \rightarrow C_{\infty}$,

$C_{\mathrm{p}} \rightarrow C_{\infty}$ as $y \rightarrow \infty$

in which the components $u, u_{\mathrm{p}}$ are the velocity components of liquid and dusty fluid along $x$ axis, respectively, $v, v_{\mathrm{p}}$ are the velocity components of liquid and dusty fluid along $y$ axis, respectively. $v$ is kinematic viscosity of the fluid, $k$ is vertex viscosity, $\rho$ is effective density of the fluid, $\sigma$ is the electrical conductivity, $B_{0}$ is magnetic field strength, $k_{1}=6 \pi \mu_{\mathrm{f}} a$ is the stokes drag constant, $\rho_{\mathrm{p}}=r s$ is density of dust particles, $m$ is velocity power law index, $s$ is number density of the dust particles, $r$ is the mass of the dust particle, $g$ is the gravitational acceleration, $\beta$ is the thermal expansion coefficient, and $j, \gamma^{*}$ and $k$ are the microinertia per unit mass, spin gradient viscosity and vertex viscosity, respectively.

Spin gradient viscosity $\gamma^{*}$ can be defined as

$\gamma^{*}=\left(\mu+\frac{k}{2}\right) j=\mu\left(1+\frac{R}{2}\right)$

where $\mu$ dynamic viscosity of the fluid, $R=\frac{k}{\mu}$ is the dimensionless viscosity ratio and is called the material parameter, and we take $j=\frac{v}{v}$ a reference length. $m_{0}$ is the boundary parameter where $T^{a}$ and $T_{\mathrm{p}}$ are the temperatures of the fluid and dust particles, respectively. $C_{\mathrm{mf}}$ is the specific heat of the dust particles, $\tau_{\mathrm{t}}$ is the thermal equilibrium time, $\tau_{v}$ is the relaxation time of the dust particle for velocity, and $C$ and $C_{\mathrm{p}}$ are the concentrations of fluid and dust particles.

Define the following similarity transformations and variable quantities:

$\zeta=\sqrt{\frac{a(\mathrm{~m}+1)}{2 v}}(x+b)^{\frac{\mathrm{m}-1}{2}} y, \quad \xi=\sqrt{\frac{2}{n+1} v a(x+b)^{\mathrm{m}+1}} h(\zeta)$,

$\xi_{\mathrm{p}}=\sqrt{\frac{2}{m+1} v a(x+b)^{\mathrm{m}+1}} H(\zeta)$,

$N=a(x+b)^{\mathrm{m}} \sqrt{\frac{a(m+1)}{2 v}}(x+b)^{\frac{\mathrm{m}-1}{2}} G(\zeta)$,

$\Theta(\zeta)=\frac{T-T_{\infty}}{T_{\mathrm{w}}-T_{\infty}}, \quad \Theta_{\mathrm{p}}(\zeta)=\frac{T_{\mathrm{p}}-T_{\infty}}{T_{\mathrm{w}}-T_{\infty}}$,

$\Phi(\zeta)=\frac{C-C_{\infty}}{C_{\mathrm{w}}-C_{\infty}}, \quad \Phi_{\mathrm{p}}(\zeta)=\frac{C_{\mathrm{p}}-C_{\infty}}{C_{\mathrm{w}}-C_{\infty}}$ 
Table 1 Comparison values of $f^{\prime \prime}(0)$ for various values of $m$

\begin{tabular}{llll}
\hline$m$ & $\begin{array}{l}\text { Animasun and } \\
\text { Sandeep [42] }\end{array}$ & Kumaran et al. [40] & Present results \\
\hline 0.1 & -0.8671009 & -0.86710092 & -0.86710093 \\
0.2 & -0.8624053 & -0.8624053 & -0.8624051 \\
0.3 & -0.8584863 & -0.8584863 & -0.8584863 \\
0.4 & -0.8481543 & -0.8481543 & -0.8481544 \\
\hline
\end{tabular}

By making use of Eq. (12), Eqs. (2)-(8) are transformed as

$$
\begin{aligned}
& (1+R) h^{\prime \prime \prime}+h h^{\prime \prime}-\frac{2 m}{m+1} h^{\prime 2} \\
& +R G^{\prime}+l \beta_{\mathrm{v}}\left(H^{\prime}-h^{\prime}\right) \\
& -\frac{2}{m+1}\left(M+\frac{1}{k_{\mathrm{p}}}\right) h^{\prime}+\mathrm{Gr}_{\mathrm{b}} \Theta \\
& +\mathrm{Gr}_{\mathrm{c}} \Phi=0 \\
& \left(1+\frac{R}{2}\right) G^{\prime \prime}+h G^{\prime}-\frac{3 m-1}{m+1} h^{\prime} G-\frac{2 R}{m+1}\left(2 G+h^{\prime \prime}\right)=0 \\
& \left(1+\frac{4}{3} \mathrm{Nr}\right) \Theta^{\prime \prime}+\frac{2}{m+1} \operatorname{Pr} h \Theta^{\prime}+\frac{2}{m+1} \operatorname{Pr} l \beta_{\mathrm{t}}\left(\Theta_{\mathrm{p}}-\Theta\right) \\
& +\frac{2}{m+1} \operatorname{Pr} l \operatorname{Ec} \beta_{\mathrm{v}}\left(H^{\prime}-h^{\prime}\right)^{2}+\operatorname{Pr} \operatorname{Ec}(1+R) h^{\prime \prime 2}+\operatorname{Pr} \operatorname{Ec} M^{2} h^{\prime 2}=0 \\
& \Phi^{\prime \prime}+\operatorname{Sc} h \Phi^{\prime}+\operatorname{Sc} \beta_{\mathrm{c}}\left(\Phi_{\mathrm{p}}-\Phi\right)+\operatorname{Sc} \operatorname{kr} \Phi=0 \\
& H H^{\prime \prime}-\frac{2 m}{m+1} H^{\prime 2}+\beta_{v}\left(h^{\prime}-H^{\prime}\right)=0 \\
& \Theta_{\mathrm{p}}^{\prime} H-\gamma \beta_{\mathrm{t}}\left(\Theta_{\mathrm{p}}-\Theta\right)=0 \\
& \Phi_{\mathrm{p}}^{\prime} H-\gamma \beta_{\mathrm{c}}\left(\Phi_{\mathrm{p}}-\Phi\right)=0 \\
& h(\alpha)=\alpha \frac{1-m}{m+1}, \quad h^{\prime}(\alpha)=1, \quad G(\alpha)=-m_{0} h^{\prime \prime}(\alpha), \\
& \Theta^{\prime}(\alpha)=-\operatorname{Bi}(1-\Theta(\alpha)), \quad \Phi(\alpha)=1 \text { at } \alpha=0 \\
& h^{\prime} \rightarrow 0, H^{\prime} \rightarrow 0, \quad H \rightarrow h, \quad G \rightarrow 0, \quad \Theta \rightarrow 0, \\
& \Theta_{\mathrm{p}} \rightarrow 0, \Phi \rightarrow 0, \Phi_{\mathrm{p}} \rightarrow 0 \text { as } \alpha \rightarrow \infty
\end{aligned}
$$

Here $\alpha=A \sqrt{\frac{a(m+1)}{2 v}}$ denotes UHSPR. To transform the equations and boundary conditions $h(\zeta-\alpha)=f(\eta)$, $G(\zeta-\alpha)=g(\eta), \quad H(\zeta-\alpha)=F(\eta), \quad \Theta(\zeta-\alpha)=\theta(\eta)$, $\Theta_{\mathrm{p}}(\zeta-\alpha)=\theta_{\mathrm{p}}(\eta)$ which gives
The governing equations are reduced to the form

$$
\begin{aligned}
& (1+R) f^{\prime \prime \prime}+f f^{\prime \prime}-\frac{2 m}{m+1} f^{\prime 2}+K g^{\prime}+l \beta_{\mathrm{v}}\left(h^{\prime}-f^{\prime}\right) \\
& -\frac{2}{m+1}\left(M+\frac{1}{k_{\mathrm{p}}}\right) f^{\prime}+\mathrm{Gr}_{\mathrm{b}} \theta+\mathrm{Gr}_{\mathrm{c}} \phi=0 \\
& \left(1+\frac{R}{2}\right) g^{\prime \prime}+f g^{\prime}-\frac{3 m-1}{m+1} f^{\prime} g-\frac{2 R}{m+1}\left(2 g+f^{\prime \prime}\right)=0
\end{aligned}
$$

$$
\begin{aligned}
(1 & \left.+\frac{4}{3} \mathrm{Nr}\right) \theta^{\prime \prime}+\frac{2}{m+1} \operatorname{Pr} f \theta^{\prime}+\frac{2}{m+1} \operatorname{Pr} l \beta_{\mathrm{t}}\left(\theta_{\mathrm{p}}-\theta\right) \\
& +\frac{2}{m+1} \operatorname{Pr} l \operatorname{Ec} \beta_{\mathrm{v}}\left(h^{\prime}-f^{\prime}\right)^{2}+\operatorname{Pr} \operatorname{Ec}(1+R) f^{\prime \prime 2}+\operatorname{Pr} \operatorname{Ec} M^{2} f^{\prime 2}=0
\end{aligned}
$$$$
\phi^{\prime \prime}+\operatorname{Sc} f \phi^{\prime}+\operatorname{Sc} \beta_{\mathrm{c}}\left(\phi_{\mathrm{p}}-\phi\right)+\operatorname{Sckr} \phi=0
$$

$F F^{\prime \prime}-F^{\prime 2}+\frac{2 m}{m+1} \beta_{\mathrm{v}}\left(f^{\prime}-F^{\prime}\right)=0$

$\theta_{\mathrm{p}}^{\prime} F-\gamma \beta_{\mathrm{t}}\left(\theta_{\mathrm{p}}-\theta\right)=0$

$\phi_{\mathrm{p}}^{\prime} F-\gamma \beta_{\mathrm{c}}\left(\phi_{\mathrm{p}}-\phi\right)=0$

and relevant boundary conditions

$f(0)=\alpha \frac{1-m}{m+1}, f^{\prime}(0)=1, \quad g(0)=-m_{0} f^{\prime \prime}(0)$,

$\theta^{\prime}(0)=-B i(1-\theta(0)), \quad \phi(0)=1$ at $\eta=0$

$f^{\prime} \rightarrow 0, f^{\prime \prime} \rightarrow 0, \quad g \rightarrow 0, \quad F^{\prime} \rightarrow 0, \quad F \rightarrow f$,

$\theta \rightarrow 0, \theta_{\mathrm{p}} \rightarrow 0, \phi \rightarrow 0, \phi_{\mathrm{p}} \rightarrow 0$ as $\eta \rightarrow \infty$

in which $R=\frac{k}{\mu}$ is material parameter, $\mathrm{Gr}_{\mathrm{b}}=\frac{g \beta\left(T_{\mathrm{w}}-T_{\infty}\right)}{a^{2}(x+b)^{2 \mathrm{~m}-1}}$ thermal buoyancy parameter, $\mathrm{Gr}_{\mathrm{c}}=\frac{g \beta *\left(C_{\mathrm{w}}-C_{\infty}\right)}{a^{2}(x+b)^{2 \mathrm{~m}-1}}$ solutal buoyancy parameter, $\operatorname{Re}_{\mathrm{x}}=u_{\mathrm{w}}(x+b) / v \quad$ Reynolds number, $M=\frac{\sigma B^{2}}{a \rho(x+b)^{\mathrm{m}-1}}$ magnetic parameter, $k_{\mathrm{p}}=\frac{a k_{\rho}}{v(x+b)^{\mathrm{m}-1}}$ porous permeability parameter $l=\frac{r s}{\rho}$ mass concentration of the dust particle, $\beta_{\mathrm{v}}=\frac{1}{a \tau_{\mathrm{v}}(x+b)^{\mathrm{m}-1}}$ the fluid particle interaction parameter for velocity, $\gamma=\frac{c_{\mathrm{m}}}{c_{\mathrm{p}}}$ specific heat coefficient, $\operatorname{Pr}=\frac{v}{\alpha}$ the Prandtl number, $\mathrm{Nr}=\frac{4 \sigma * T_{\infty}^{3}}{3 k_{\mathrm{f}} k^{*}}$ radiation parameter, $\mathrm{Ec}=\frac{u_{\mathrm{w}}^{2}}{c_{\mathrm{p}}\left(T_{\mathrm{w}}-T_{\infty}\right)}$ the Eckert number, $\beta_{\mathrm{t}}=\frac{1}{a \tau_{\mathrm{t}}(x+b)^{\mathrm{m}-1}}$ the fluid particle interaction parameter for temperature, $\mathrm{Sc}=\frac{v}{D_{\mathrm{m}}}$ Schmidt number, $\mathrm{kr}=\frac{\mathrm{Kr} *\left(C_{\mathrm{w}}-C_{\infty}\right)}{\mathrm{c}}$ chemical reaction parameter, $\beta_{\mathrm{c}}=\frac{1}{a \tau_{\mathrm{c}}(x+b)^{\mathrm{m}-1}}$ the fluid particle interaction parameter for concentration, and $\mathrm{Bi}=\frac{h_{\mathrm{f}}}{k_{\mathrm{f}}} \sqrt{\frac{v}{a}}(x+b)^{\left(\frac{\mathrm{m}-1}{2}\right)}$ the Biot number. 

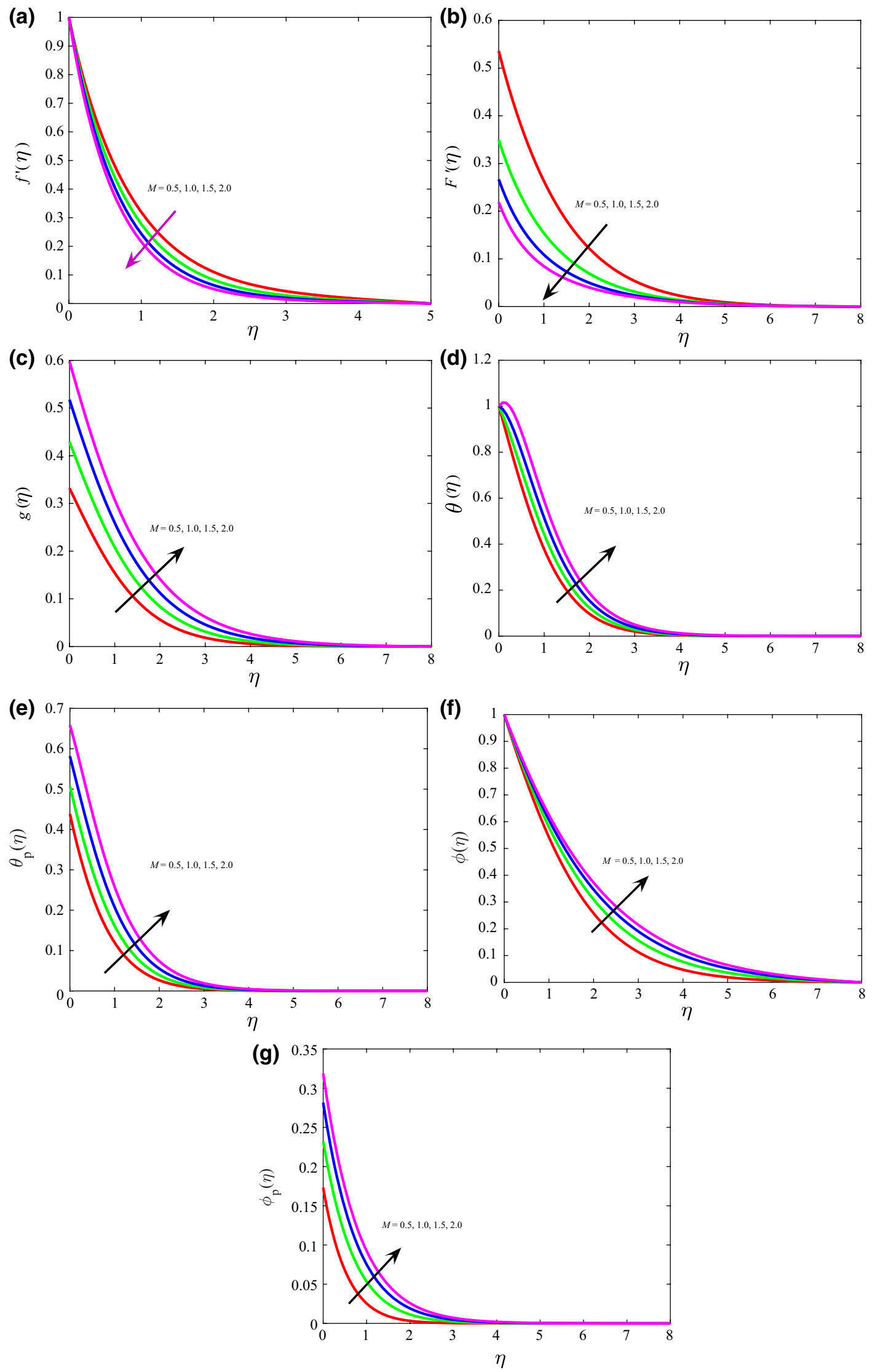
4Fig. 2 Impact of Magnetic parameter on a velocity profile. b Dust phase velocity profile. c Angular velocity profile. d Temperature profile. e Dust phase temperature profile. f Concentration profile $\phi(\eta)$. $\mathbf{g}$ Dust phase concentration profile

For physical quantities of engineering interest [39, 40]:

$$
\begin{aligned}
& C_{\mathrm{fx}}=\frac{\tau_{\mathrm{w}}}{\rho u_{\mathrm{w}}^{2}}\left(\frac{m+1}{2}\right)^{-0.5}, \mathrm{Nu}_{\mathrm{x}}=\frac{(x+b) q_{\mathrm{w}}}{k_{\mathrm{f}}\left(T_{\mathrm{w}}-T_{\infty}\right)}\left(\frac{m+1}{2}\right)^{-0.5}, \\
& \mathrm{Sh}_{\mathrm{x}}=\frac{(x+b) j_{\mathrm{w}}}{k_{\mathrm{f}}\left(C_{\mathrm{w}}-C_{\infty}\right)}\left(\frac{m+1}{2}\right)^{-0.5}
\end{aligned}
$$

where $\tau_{\mathrm{w}}$ the surface shear stress $q_{\mathrm{w}}$ is the surface heat flux and $j_{\mathrm{w}}$ is the mass flux; they are given by:

$\tau_{\mathrm{w}}=\mu\left(\frac{\partial u}{\partial y}\right)_{\eta=0}, \quad q_{\mathrm{w}}=-k\left(\frac{\partial T}{\partial y}\right)_{\eta=0}$ and $j_{\mathrm{w}}=-D_{\mathrm{m}}\left(\frac{\partial C}{\partial y}\right)_{\eta=0}$
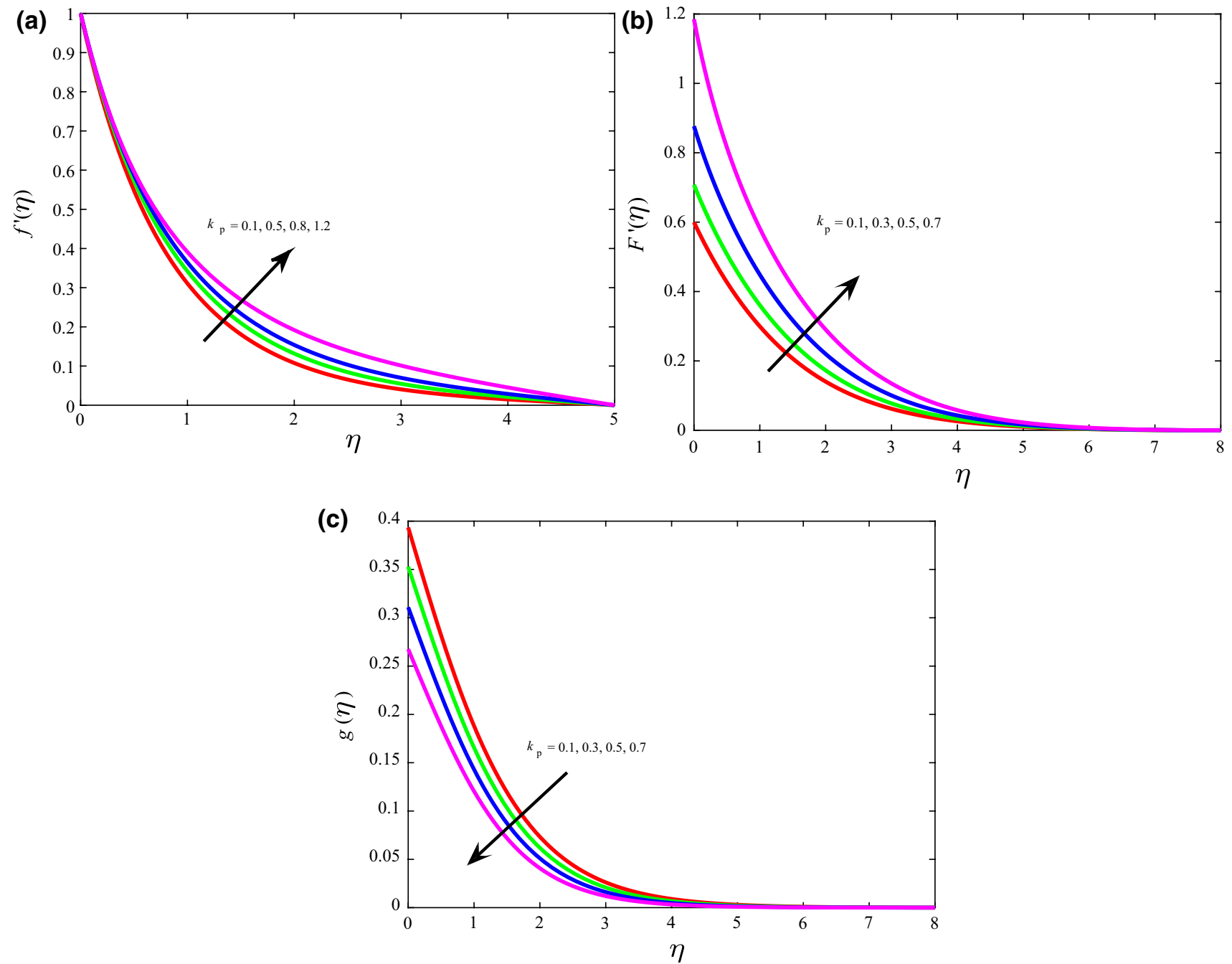

Fig. 3 Impact of permeability parameter on a velocity profile. b Dust phase velocity profile. $\mathbf{c}$ Angular velocity profile 

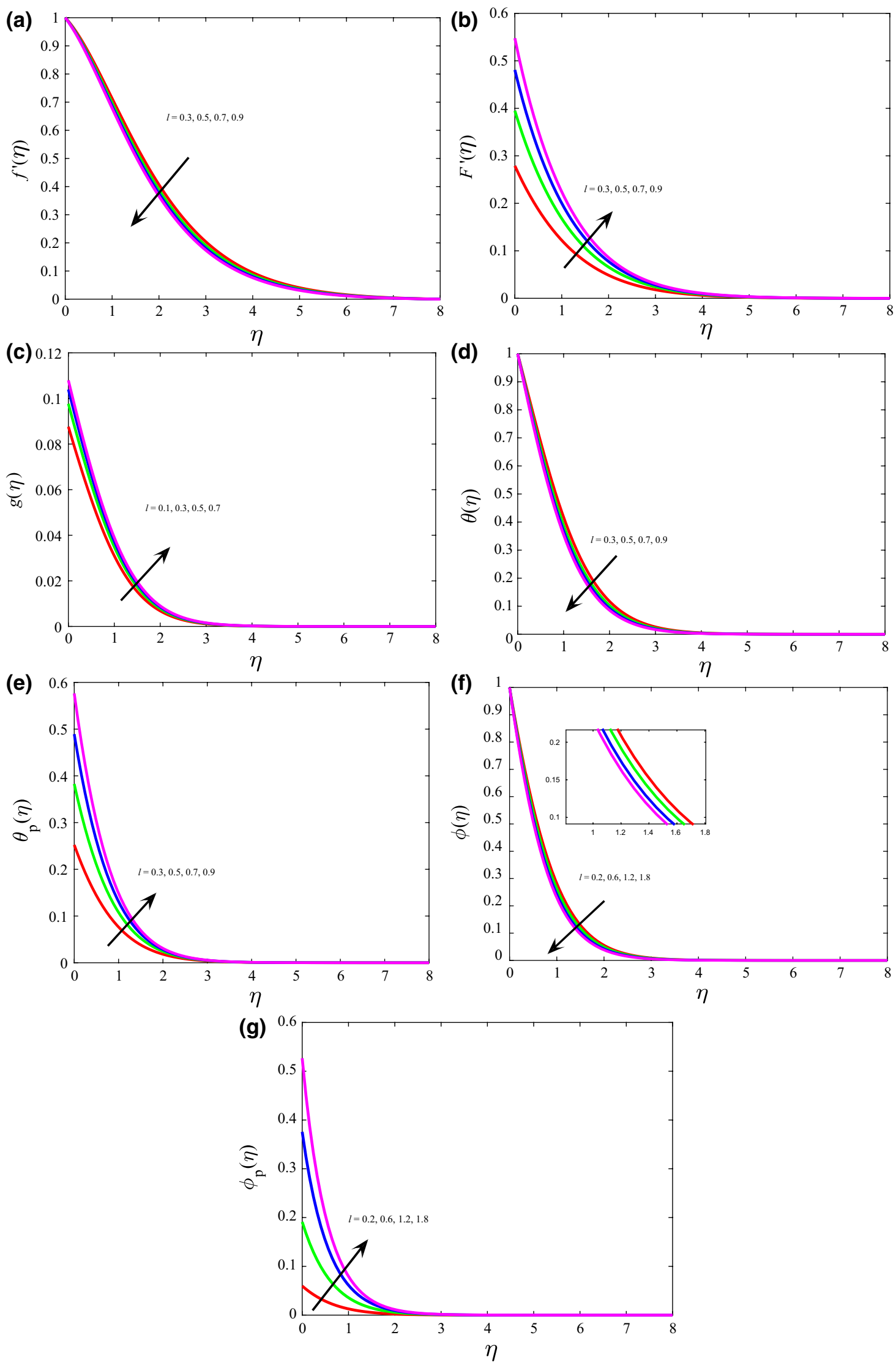
४Fig. 4 Impact of mass concentration of the dust particle on a velocity profile. b Dust phase velocity profile. c Dust particle on angular velocity profile. d Dust particle on temperature profile. e Dust particle on dust phase temperature profile. $\mathbf{f}$ Dust particle on concentration profile. $\mathbf{g}$ Dust particle on dust phase concentration profile

$$
\begin{aligned}
& f=f(1), \quad f^{\prime}=f(2), \quad f^{\prime}=f(3) \\
& f^{\prime \prime}=-\frac{1}{(1+R)}\left[\begin{array}{c}
f(1) f(3)-\frac{2 m}{m+1}[f(2)]^{2}+K f(5)+l \beta_{\mathrm{v}}(f(11)-f(2)) \\
-\frac{2}{m+1}\left(M+\frac{1}{k_{\mathrm{p}}}\right) f(2)+\mathrm{Gr}_{\mathrm{b}} f(6)+\mathrm{Gr}_{\mathrm{c}} f(8)
\end{array}\right]
\end{aligned}
$$

differential Eqs. (21)-(27) are converted to first-order differential equations, by using the following procedure
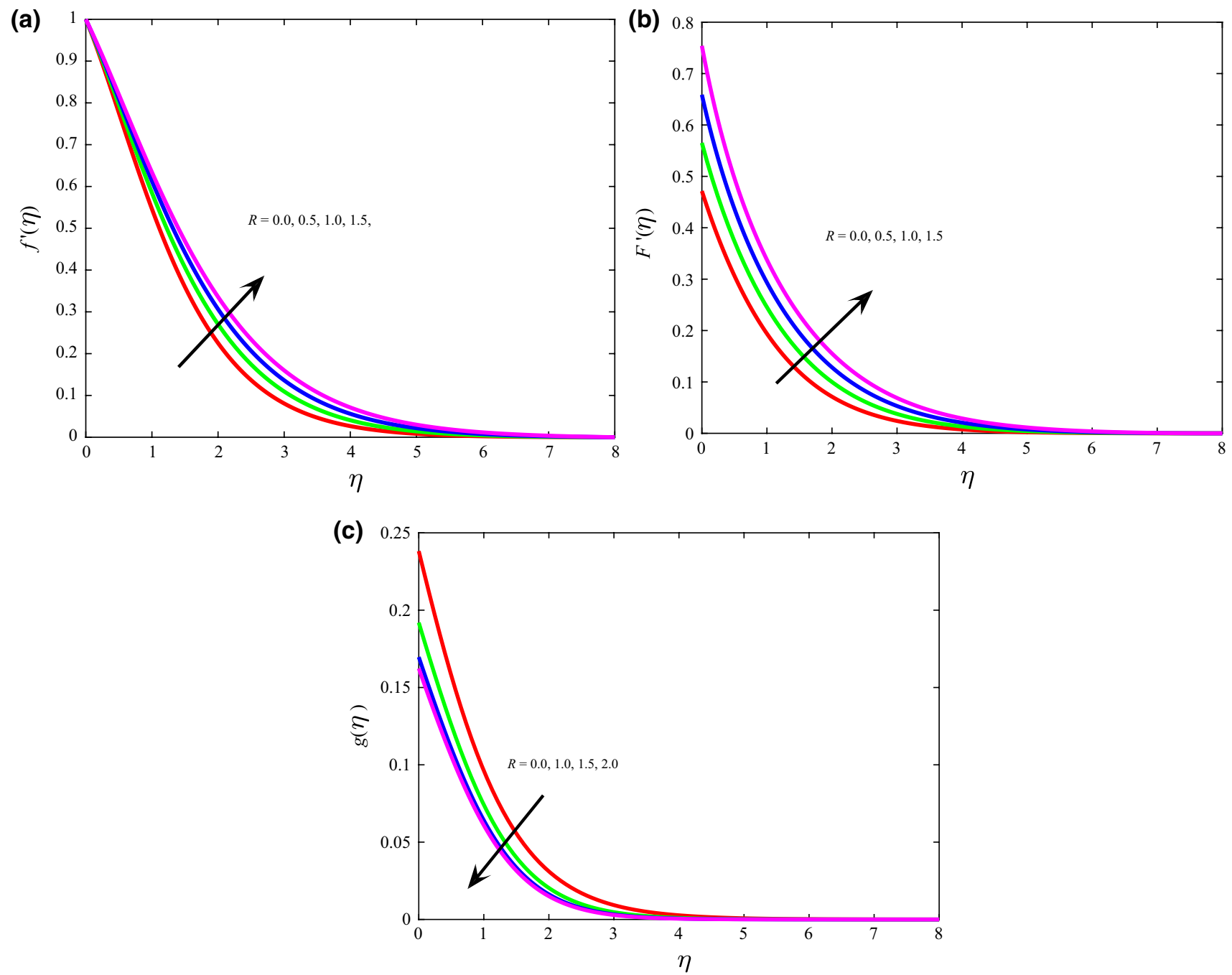

Fig. 5 Impact of material parameter on a velocity profile. b Dust phase velocity profile. $\mathbf{c}$ Angular velocity profile 

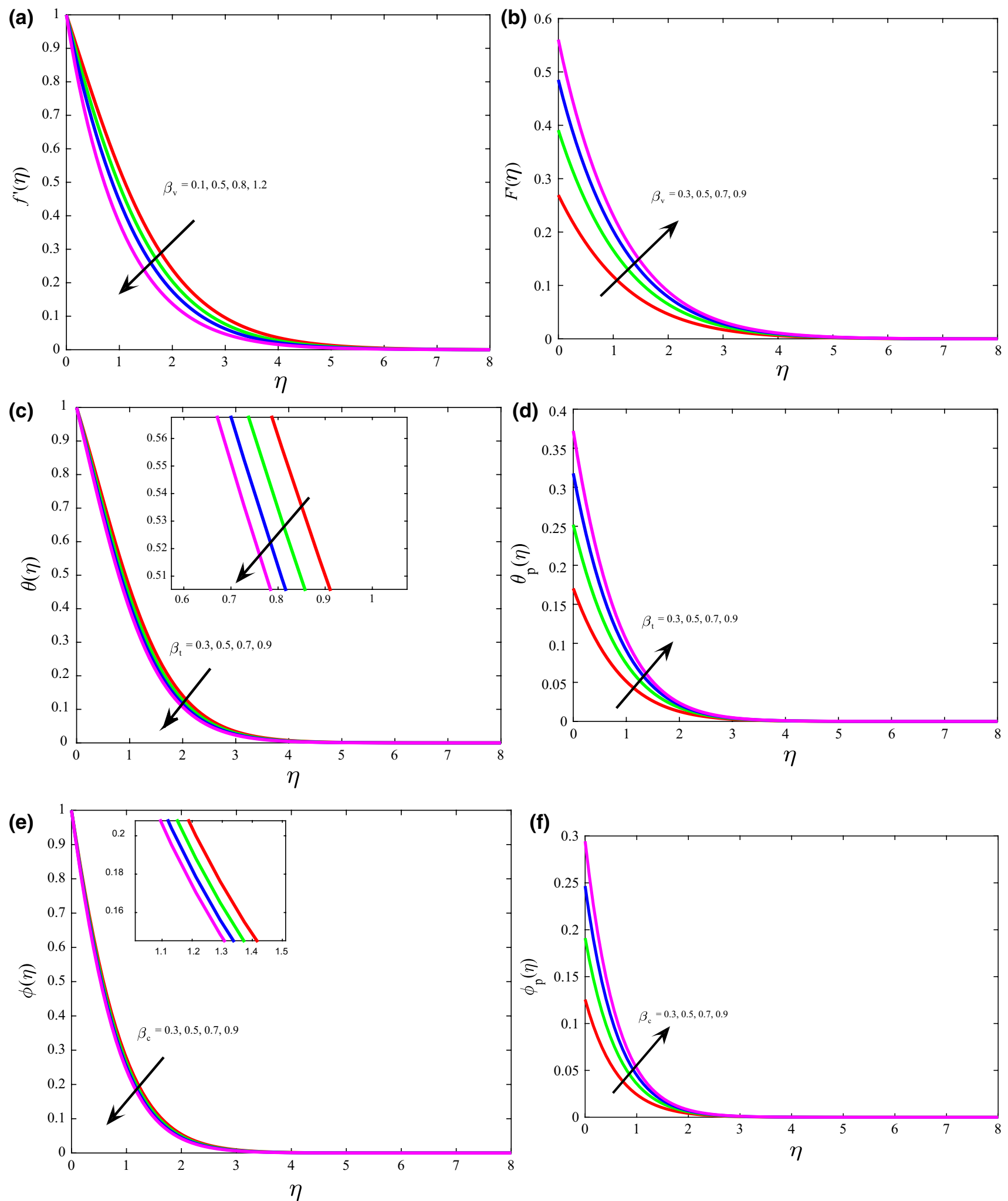

Fig. 6 Impact of fluid particle interaction parameter for velocity on a velocity profile. b Dust phase velocity profile. $\mathbf{c}$ Temperature on temperature profile. $\mathbf{d}$ Temperature on dust phase temperature profile. e Concentration on concentration profile. $\mathbf{f}$ Dust phase concentration profile 

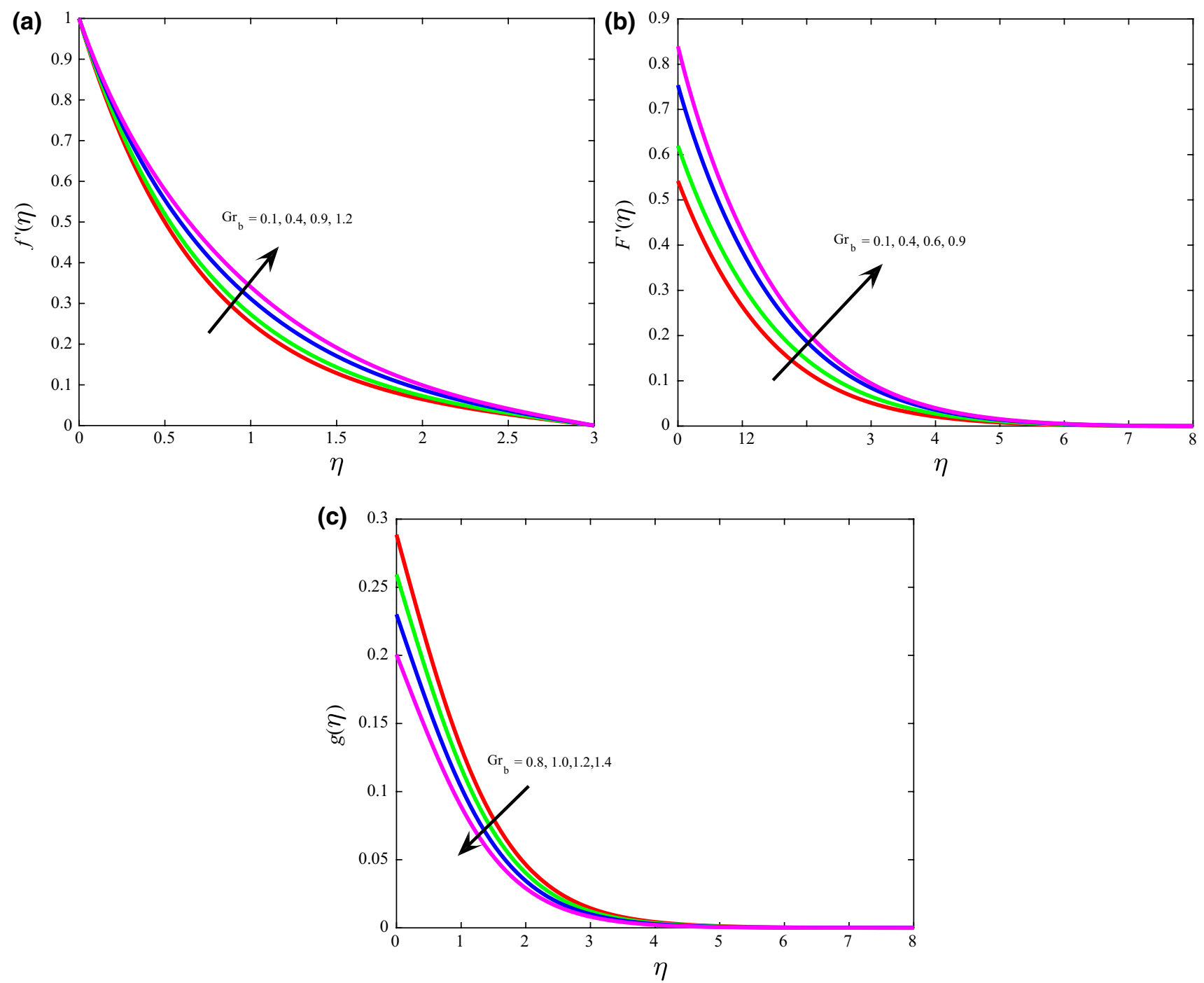

Fig. 7 Impact of thermal buoyancy parameter on a velocity profile. b Dust phase velocity profile. $\mathbf{c}$ Angular velocity profile

$g=f(4), \quad g^{\prime}=f(5)$
$g^{\prime \prime}=-\frac{1}{\left(1+\frac{R}{2}\right)}\left[f(1) f(5)-\frac{3 m-1}{m+1} f(2) f(4)-\frac{2 R}{m+1}(2 f(4)+f(3))\right]$

$\theta=f(6), \quad \theta^{\prime}=f(7)$

$\theta^{\prime}=-\frac{1}{\left(1+\frac{4}{3} \mathrm{Nr}\right)}\left[\begin{array}{c}\frac{2}{m+1} \operatorname{Pr} f(1) f(7)+\frac{2}{m+1} \operatorname{Pr} l \beta_{\mathrm{t}}(f(12)-f(6)) \\ +\frac{2}{m+1} \operatorname{Pr} l \operatorname{Ec} \beta_{\mathrm{v}}(f(11)-f(2))^{2}+\operatorname{Pr} \operatorname{Ec}(1+R)[f(3)]^{2} \\ +\operatorname{Pr} \operatorname{Ec} M^{2}[f(2)]^{2}\end{array}\right]$

$F=f(10), \quad F^{\prime}=f(11)$

$F^{\prime \prime}=\frac{1}{f(10)}\left[[f(11)]^{2}-\frac{2 m}{m+1} \beta_{\mathrm{v}}(f(2)-f(11))\right]$ $\phi=f(8), \quad \phi^{\prime}=f(9)$

$\phi^{\prime \prime}=-\left[\operatorname{Sc} f(1) f(9)+\operatorname{Sc} \beta_{\mathrm{c}}(f(13)-f(8))+\operatorname{Sckr} f(8)\right]$

$$
\begin{aligned}
& \theta_{\mathrm{p}}=f(12) \\
& \theta_{\mathrm{p}}^{\prime}=\frac{1}{f(10)} \gamma \beta_{\mathrm{t}}(f(12)-f(6))
\end{aligned}
$$



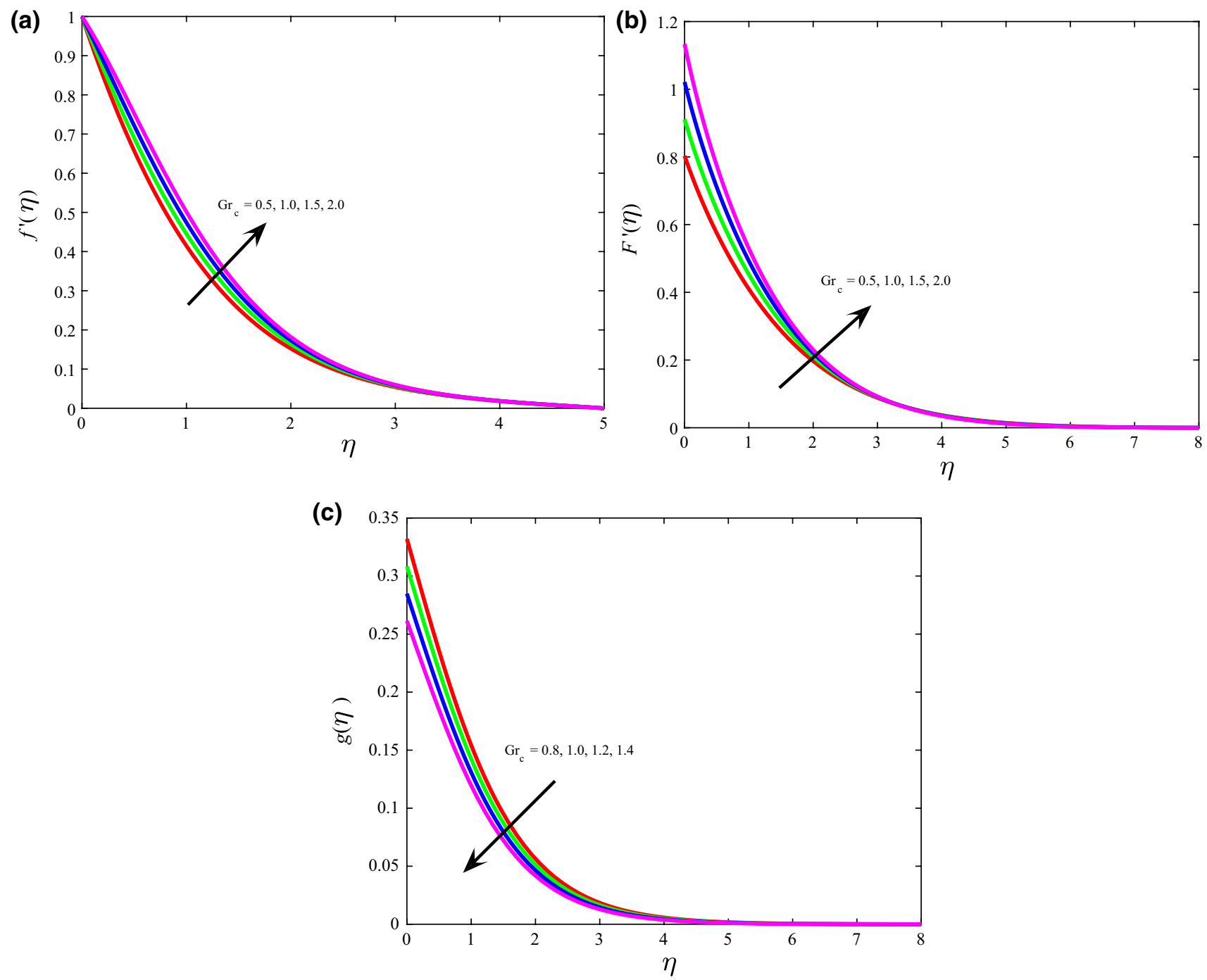

Fig. 8 Impact of solutal buoyancy parameter on a velocity profile. b Dust phase velocity profile. $\mathbf{c}$ Angular velocity profile

$\phi_{\mathrm{p}}=f(13)$

$\phi_{\mathrm{p}}^{\prime}=\frac{1}{f(10)} \gamma \beta_{\mathrm{c}}(f(13)-f(8))$

and relevant boundary conditions fourth-order method with the successive iterative step size 0.01 . Table 1 represents the comparison of $f^{\prime \prime}(0)$ for various values of $m$ with existing work. It is concluded that the present results are in good agreement with the previous studies.

$$
\begin{aligned}
& f(1)=\alpha \frac{1-m}{m+1}, f(2)=1, f(4)=-m_{0} f(3), f(7)=-\operatorname{Bi}(1-f(6)), f(8)=1 \text { at } \eta=0 \\
& f(2) \rightarrow 0, f(3) \rightarrow 0, f(4) \rightarrow 0, f(11) \rightarrow 0, f(10) \rightarrow f(1),\} \\
& f(6) \rightarrow 0, f(12) \rightarrow 0, f(8) \rightarrow 0, f(12) \rightarrow 0 \\
& \text { as } \eta \rightarrow \infty
\end{aligned}
$$

To solve Eqs. (29)-(35), we guess the values of $y_{4}, y_{6}$, $y_{8}, y_{9}, y_{11}$ and $y_{12}$ which are not given at the initial conditions. Once all initial conditions are found, then we solve Eqs. (29)-(35) which are integrated by using $R-K$

\section{Graphical discussion}

For summarizing the effects of several flow quantities $f^{\prime}, g, F^{\prime}, \theta, \theta_{\mathrm{p}}, \phi$ and $\phi_{\mathrm{p}}$. Here we fix the nondimensional variables for numerical calculations as 

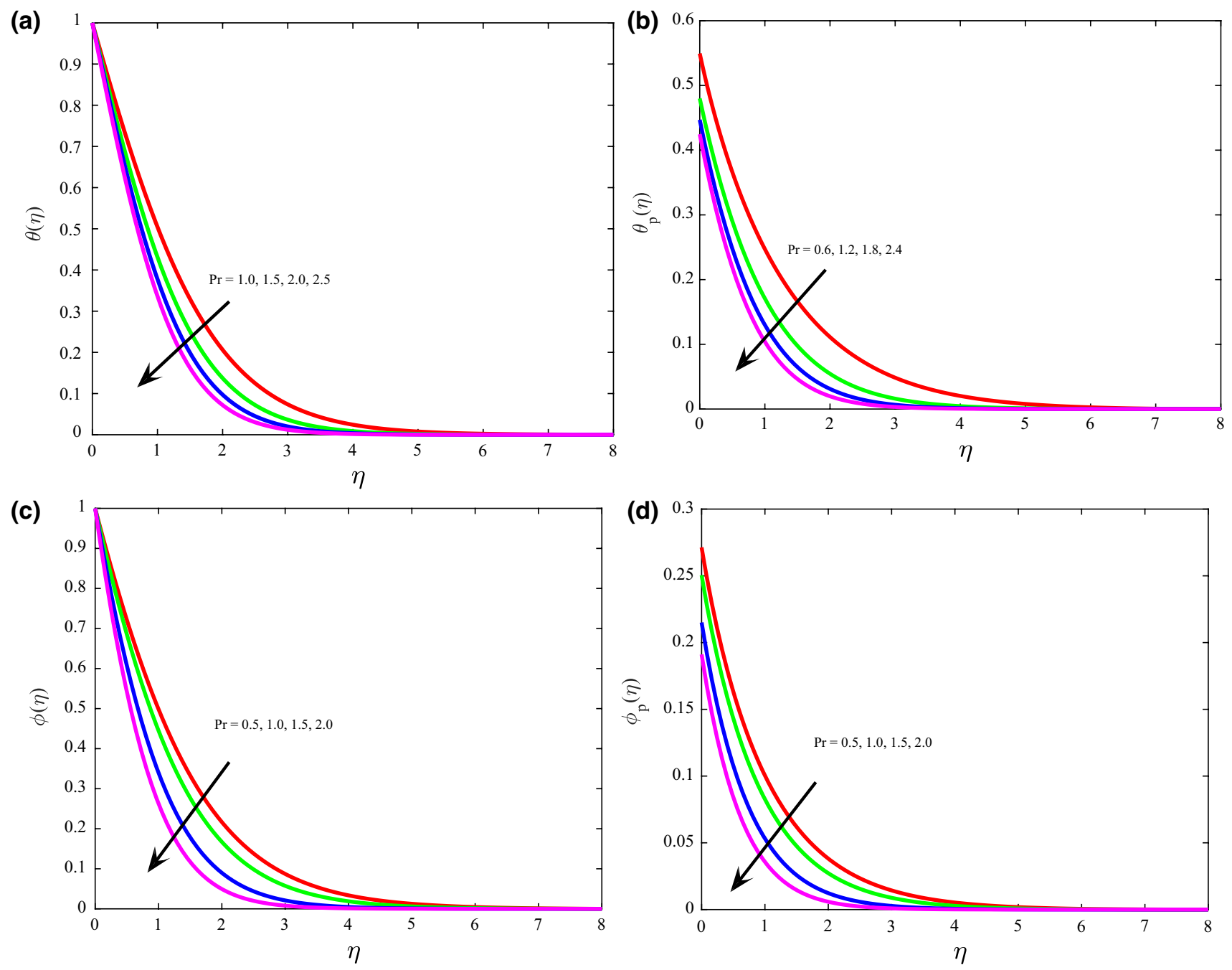

Fig. 9 Impact of Prandtl number on a temperature profile. b Dust phase temperature profile. $\mathbf{c}$ Concentration profile. $\mathbf{d}$ Dust phase concentration profile

$M=0.5, R=1.2, \mathrm{Ec}=0.5, \operatorname{Pr}=0.6, \mathrm{Gr}_{\mathrm{b}}=0.8, \mathrm{Gr}=0.8_{\mathrm{c}}$, $\alpha=0.5, l=1.2, m=0.6, \mathrm{kr}=0.3, \mathrm{kp}=0.4, \beta_{\mathrm{v}} \boldsymbol{\beta}_{\mathrm{t}}=1.2=\beta_{\mathrm{c}}$, $\mathrm{Nr}=0.5$; throughout the study these values have been not changed.

Figure 2a-g displays the effects of magnetic parameter $M$ on $f^{\prime}, g, F^{\prime}, \theta, \theta_{\mathrm{p}}, \phi$ and $\phi_{\mathrm{p}}$. We can reveal that velocity diminishes for both the phases with boost up values of $M$ and the opposite behaviour is seen in other profiles. This declares that magnetic field repel the liquid transfer due to boost up values in $M$ tends to increment in Lorentz force, which counter the fluid flow. This slows down the motion of the fluid. Magnetic field can regulate boundary layer division. This is due to the fact that magnetic field builds a resistance force on the fluid in the boundary layer. This results the sink in the thinness of the thermal and solutal boundary layer. Moreover, impact of $M$ on dusty phase flow in $F^{\prime}, \theta_{\mathrm{p}}$ and $\phi_{\mathrm{p}}$ is almost enclosed within the dynamic region [0, 0.23], [0.41, 0.69], [0.17, 0.37].

Figure $3 \mathrm{a}-\mathrm{c}$ demonstrates the impacts of permeability parameter $k_{\mathrm{p}}$ on velocity and angular velocity profiles for both the phases. It is apparently that the occurrence of a porous medium effect higher restraint to the liquid flow which in twirl, slows its motion. Consequently, with escalating permeability parameter, the resistance to the fluid motion also increases. This affects the fluid velocity, dusty fluid velocity increases, and opposite behaviour is seen in angular velocity. 

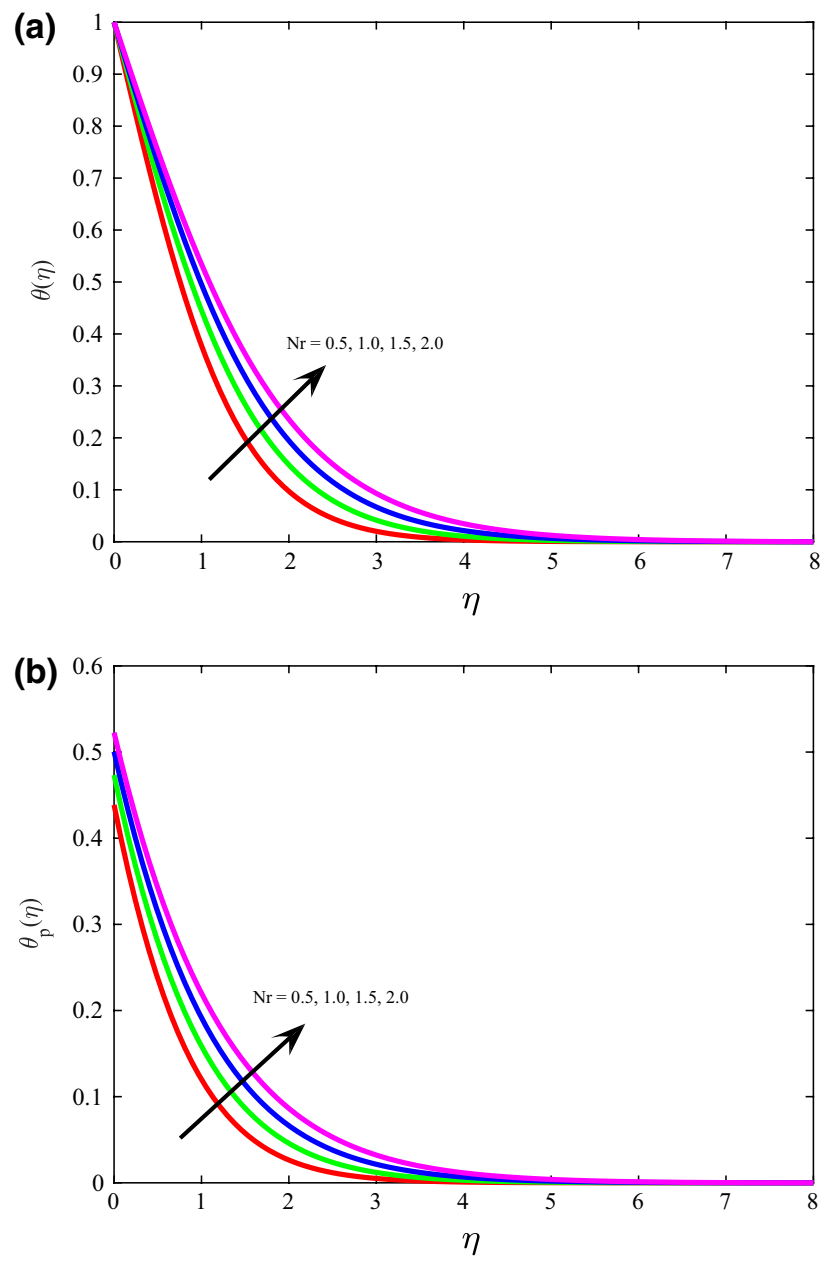

Fig. 10 Impact of radiation parameter on a temperature profile. b Dust phase temperature profile

Figure $4 \mathrm{a}-\mathrm{g}$ is aimed to the impact of mass concentration of the dust particle parameter $l$ on velocity, angular velocity, thermal and species profiles for both the phases. The profiles $f^{\prime}, g, F^{\prime}, \theta, \theta_{\mathrm{p}}, \phi$ and $\phi_{\mathrm{p}}$ decline with boost up values of $l$. Physically, the drag force between the liquid and dusty phase is intensified by escalating the mass concentration of dust particles. As a result, the momentum, thermal and solutal of the fluid delayed rapidly. Consequently, the particle phase momentum, thermal and solutal are lower too, whereas the particle phase is being dragged along by the fluid. Further, the more dust particles suck up the heat from the fluid when they come into contact, but the solitary particles will receive less power from the liquid phase.

The impact of material parameter $R$ on velocity and angular velocity is illuminated in Fig. 5a-c. Figure $5 \mathrm{a}-\mathrm{b}$ can be seen that with escalating values of $R$, the velocity profile increases for both the fluids (fluid phase, dust phase). Due
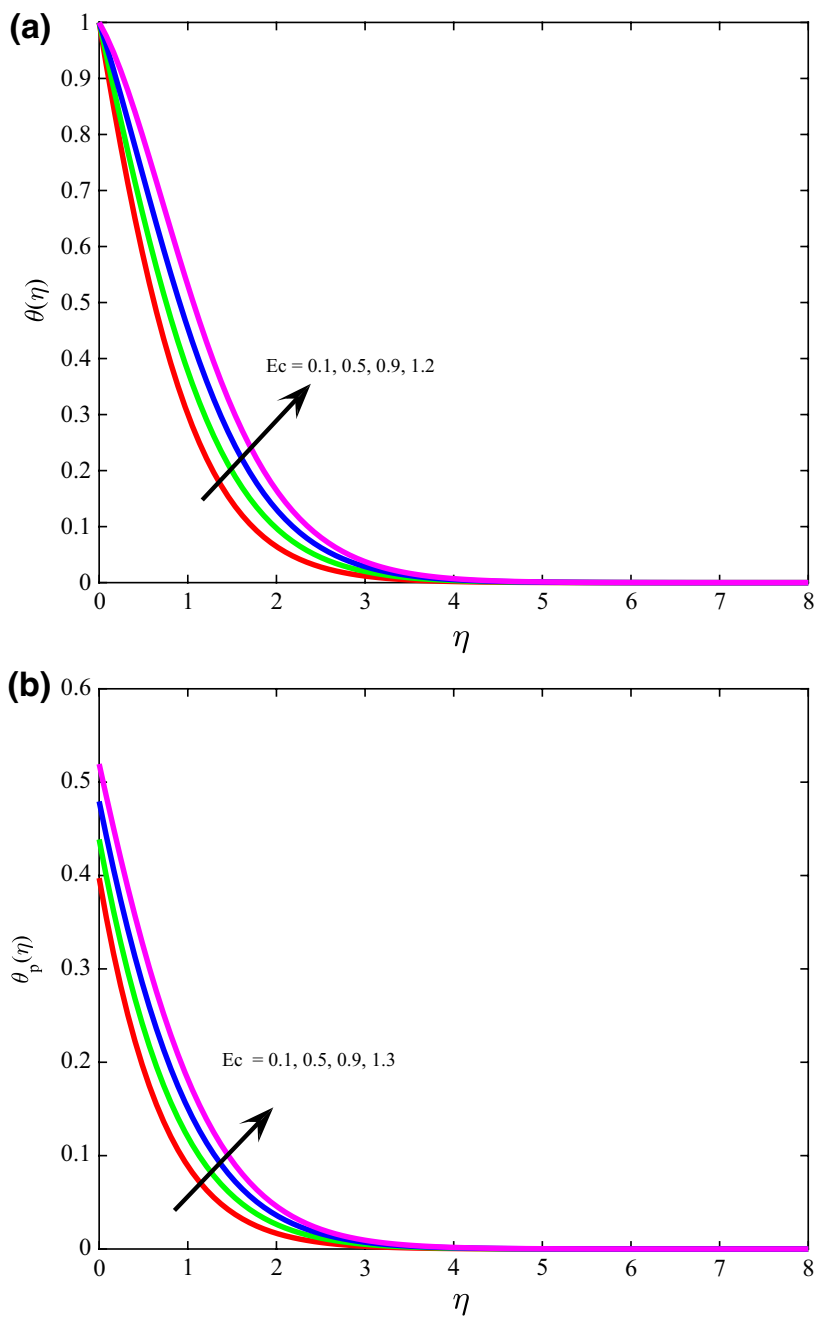

Fig. 11 Impact of Eckert number on a temperature profile. b Dust phase temperature profile

to a reason that escalating values of $R$ which corresponds to low viscosity and magnifies the velocity. And the reverse behaviour is seen in Fig. 5c. Material parameter denotes the fragment of vertex viscosity to dynamic viscosity. The dynamic viscosity declines by escalating values of $R$.

Figure 6a-f illustrates to see the variations of $\beta_{\mathrm{v}}, \beta_{\mathrm{t}}$ and $\beta_{\mathrm{c}}$ on the dimensionless distributions in the both the phases. Figure $6 \mathrm{a}, \mathrm{c}$, e shows that the $f^{\prime}(\eta), \theta(\eta)$ and $\phi(\eta)$ functions decline in micropolar fluid phase with escalating values of $\beta_{\mathrm{v}}, \beta_{\mathrm{t}}$ and $\beta_{\mathrm{c}}$, respectively. But an opposite behaviour is seen in particle phases, and particle phase velocity, temperature, concentration are almost covered within the dynamic region [0.28, 0.58], [0.18, 0.38], [0.15, 0.29] (see Fig. 6b, d, f).

The effect of thermal Grashof number is presented in Fig. 7a-c. Figure $7 \mathrm{a}, \mathrm{b}$ exhibits the fluid phase and dust 
(a)

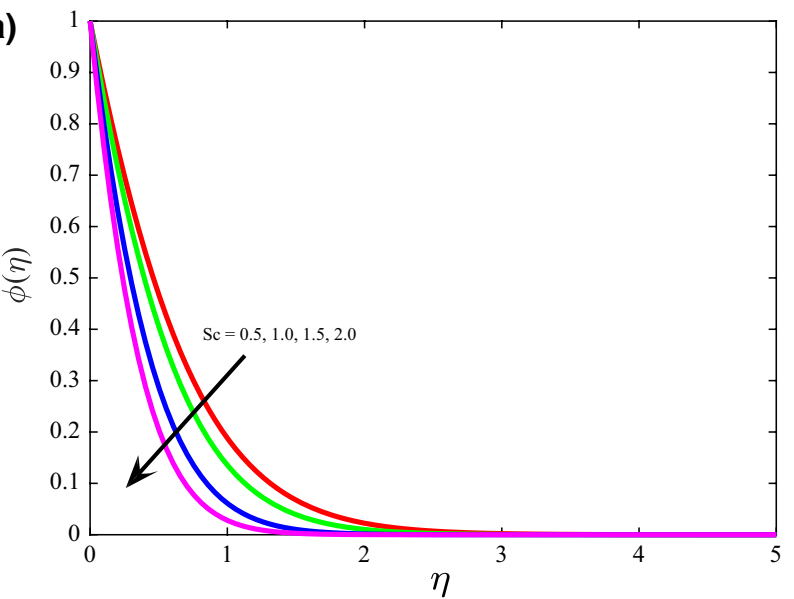

(b)

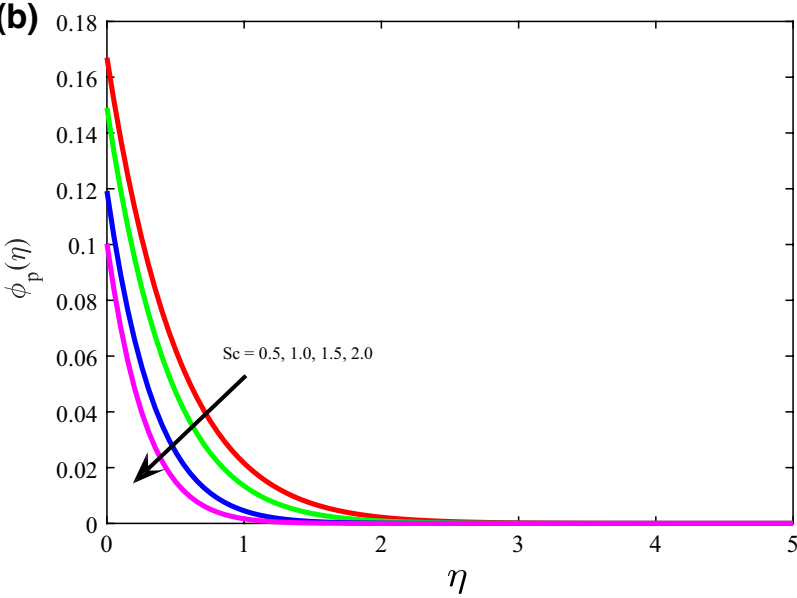

Fig. 12 Impact of Schmidt number on a concentration profile. b Dust phase concentration profile

phase velocity profiles for different values of $\mathrm{Gr}_{\mathrm{b}}$. We observed a significant increase in $f^{\prime}(\eta), F^{\prime}(\eta)$ when $\mathrm{Gr}_{\mathrm{b}}$ rises. Reverse trend is seen in Fig. 7c. We noticed as $g(\eta)$ diminishes near UHSPR for accumulating values of $\mathrm{Gr}_{\mathrm{b}}$. Figure $8 \mathrm{a}, \mathrm{b}$ declares the fluid phase and dust phase velocity profiles for different values of $\mathrm{Gr}_{\mathrm{c}}$. We observed that $f^{\prime}(\eta), F^{\prime}(\eta)$ diminishes near the surface when $\mathrm{Gr}_{\mathrm{c}}$ advances. Figure $8 \mathrm{c}$ represents the effect of $\mathrm{Gr}_{\mathrm{c}}$ on $g(\eta)$. We noticed as $g(\eta)$ diminishes near upper horizontal sphere of paraboloid revolution for accumulating values of $\mathrm{Gr}_{\mathrm{c}}$.

The impact of Prandtl number Pr is depicted in Fig. 9a-d. An escalating value of Pr temperature and concentration profiles diminishes for both the fluids. Prandtl number is a material property. It varies from fluid to fluid. Prandtl number is large when thermal conductivity is small and viscosity is large. Prandtl number's growing values reduce the thermal diffusivity, and as a result the heat flows below the fluid and the thermal boundary layer thickness reduces with increase in the values of Prandtl number. Furthermore, the effects of
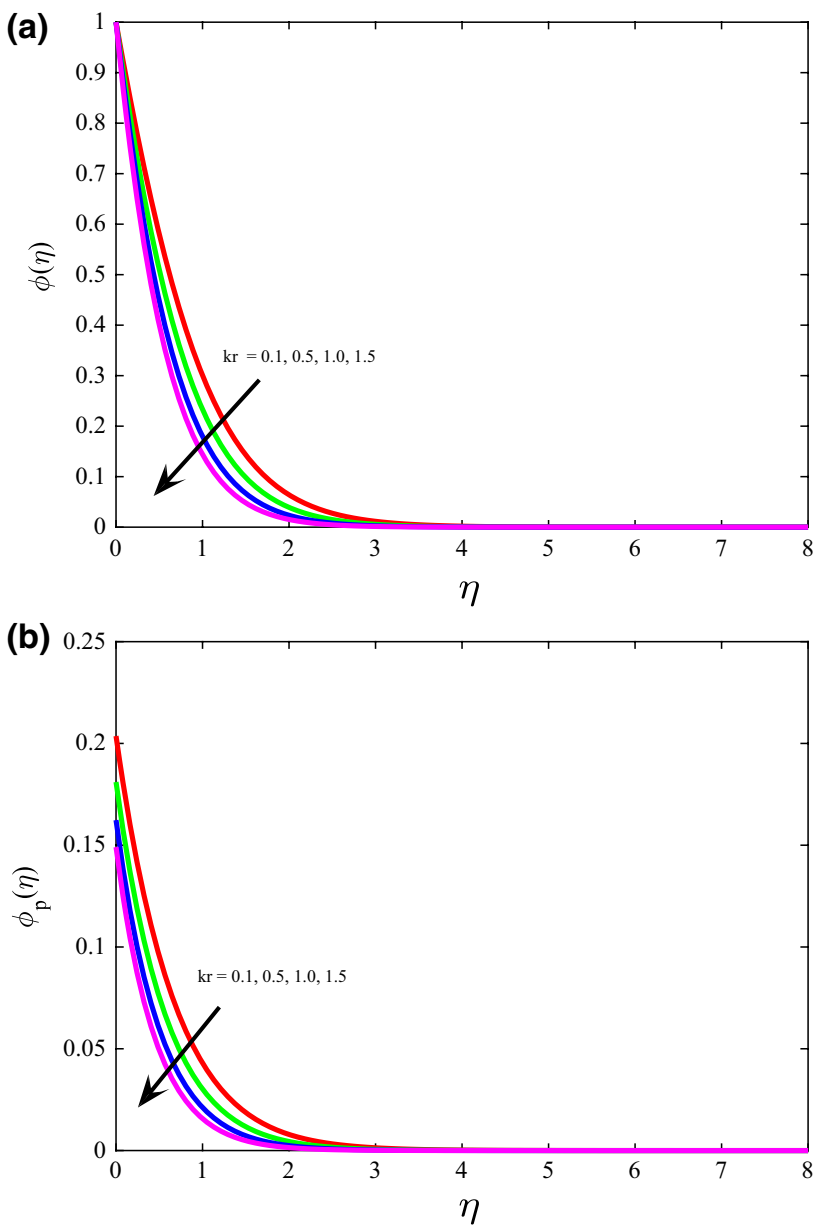

Fig. 13 Impact of chemical reaction parameter on a concentration profile. b Dust phase concentration profile

Pr on particle phase temperature and species phase temperature are almost covered within the dynamic region $[0.42$, 0.55], [0.18, 0.28].

The impact of radiation parameter $\mathrm{Nr}$ is illustrated in Fig. 10a, b. For boost up values of $\mathrm{Nr}$, it shows an increase in temperature for both the fluids. Due to the reason that intensifying radiation parameter releases the heat energy to the flow, it helps to enhance the temperature profiles. Thus, $\mathrm{Nr}$ impacts play a main role in amplifying the rate of heat transfer. This performance is qualitatively agreed with earlier published work [19].

Figure 11a, b shows the temperature profile for different values of Ec. Eckert number defines the ratio of the adjective mass transfer to the heat dissipation. It is the ratio of the kinetic energy to the enthalpy (or the dynamic temperature to the temperature) driving force for heat transfer. The results show the impact of escalating values of Ec; the temperature increases for both the fluids. 

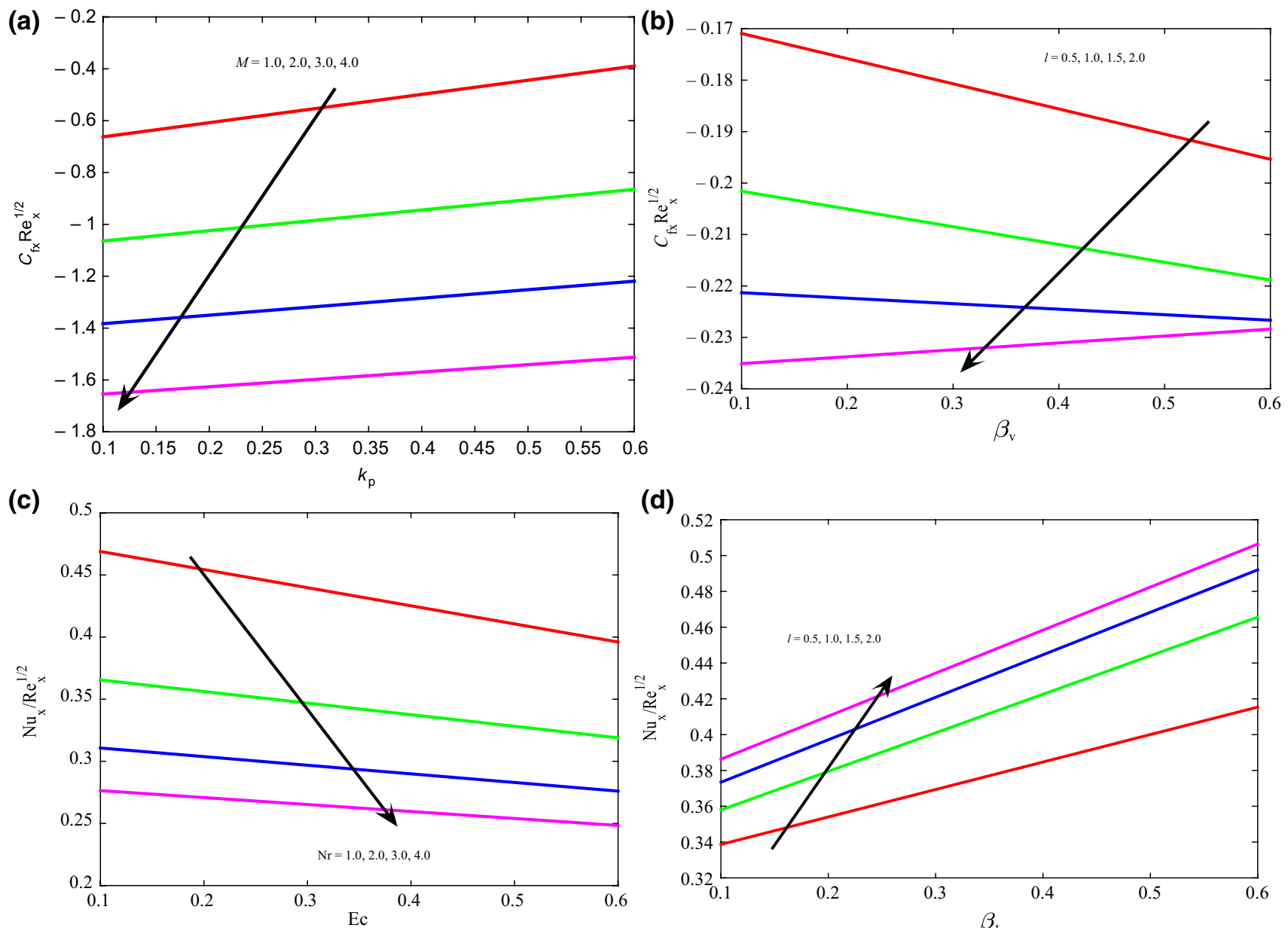

(d)
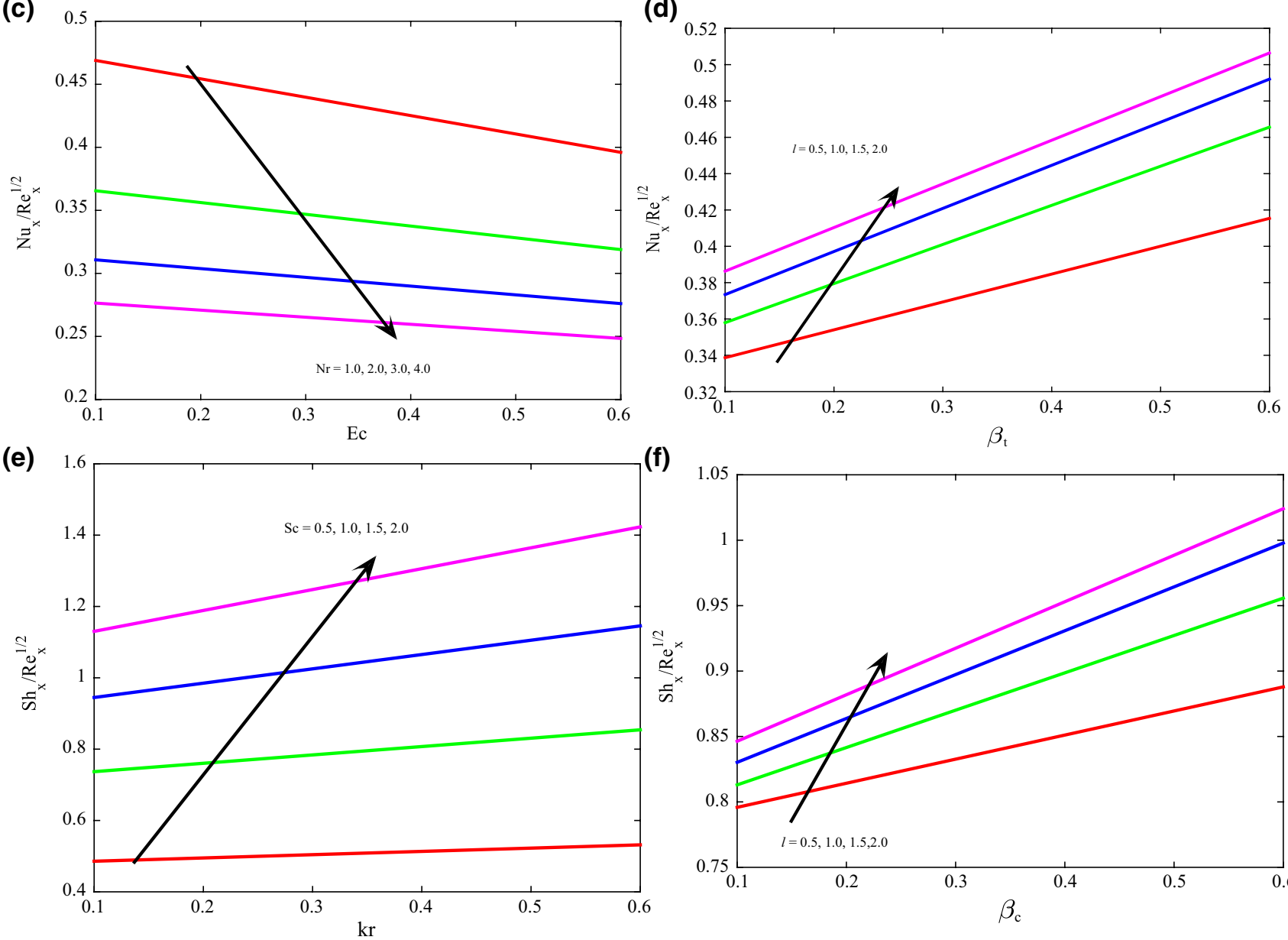

(f)

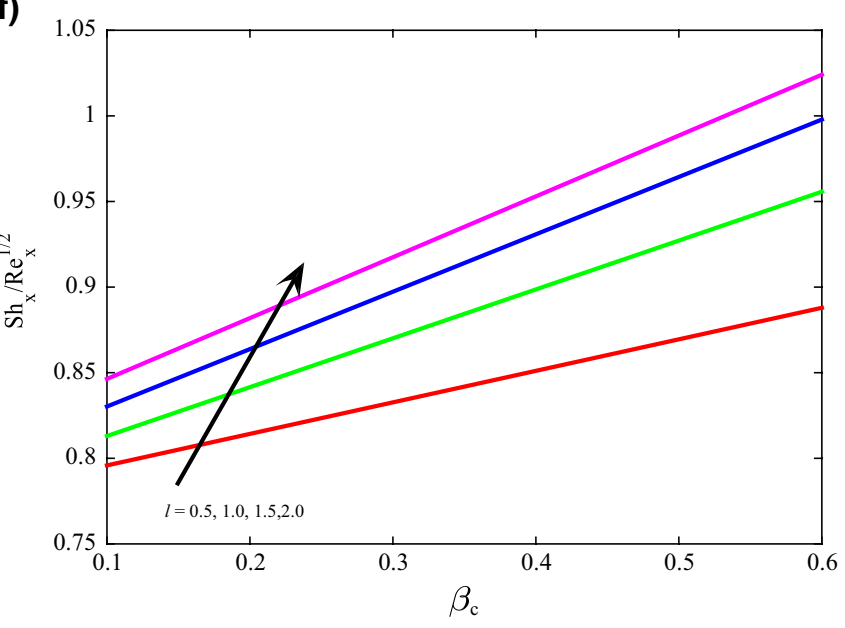

Fig. 14 a Impact of permeability parameter versus magnetic parameter on $C f_{\mathrm{x}}$. b Impact of fluid particle interaction parameter for velocity versus mass concentration of the dust particle on $C f_{\mathrm{x}}$. $\mathbf{c}$ Impact of Eckert number versus radiation parameter on $\mathrm{Nu}_{\mathrm{x}}$. d Impact of fluid particle interaction parameter for temperature versus mass concentra-

tion of the dust particle on $\mathrm{Nu}_{\mathrm{x}}$. e Impact of chemical reaction parameter versus $\mathrm{Schmidt}$ number on $\mathrm{Sh}_{\mathrm{x}}$. $\mathbf{f}$ Impact of fluid particle interaction parameter for concentration versus mass concentration of the dust particle on $\mathrm{Sh}_{\mathrm{x}}$ 


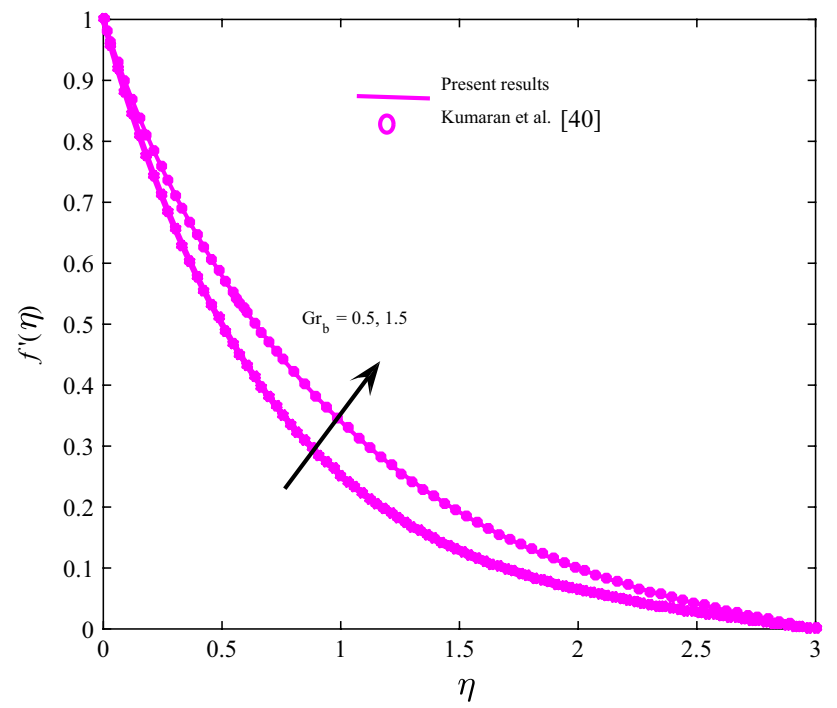

Fig. 15 Impact of thermal buoyancy parameter on velocity profile

Figure $12 \mathrm{a}, \mathrm{b}$ shows the impact of Sc on $\phi(\eta), \phi_{\mathrm{p}}(\eta)$. For mount up values of $\mathrm{Sc}$, the concentration rises for both the phases. Schmidt number is defined as the ratio of momentum diffusivity and mass diffusivity. Schmidt number is used to describe fluid flows in which there are simultaneous momentum and mass diffusion convection processes. Schmidt number relates the relative thickness of the hydrodynamic layer and mass transfer boundary layer. Raise of the values indicates strong molecular motions which rise the fluid temperature. In case of higher values, shorter penetration depth of temperature occurs. It exhibits that the dimensionless concentration profile declines as the Schmidt number rises. The impact of chemical reaction parameter $\mathrm{kr}$ on $\phi(\eta), \phi_{\mathrm{p}}(\eta)$ is drawn in Fig. 13a, b; the concentration and boundary layer thickness diminish for escalating values of $\mathrm{kr}$.

Figure $14 \mathrm{a}, \mathrm{b}$ is sketched to interrogate $C f_{\mathrm{x}}$ for contrasting values of $M, k_{\mathrm{p}}, \beta_{\mathrm{v}}$ and $l$. It conveys that due to the rise in $M, k_{\mathrm{p}}, \beta_{\mathrm{v}}$ and $l$ decline the $C f_{\mathrm{x}}$. Effect of $\mathrm{Nr}, \mathrm{Ec}, \beta_{\mathrm{t}}$ and $l$ parameters on $\mathrm{Nu}_{\mathrm{x}}$ is sketched in Fig. $14 \mathrm{c}$, d. It is clearly observed that rising values of $\mathrm{Nr}$ and Ec declines the $\mathrm{Nu}_{\mathrm{x}}$, and opposite trend is seen in $\beta_{\mathrm{t}}$ and $l$. Fluctuation in $\mathrm{Sh}_{\mathrm{x}}$ for $\mathrm{kr}, \mathrm{Sc}, \beta_{\mathrm{c}}$ and $l$ can be seen in Fig. 14e. Rising values of $\mathrm{kr}$, $\mathrm{Sc}, \beta_{\mathrm{c}}$ and $l \mathrm{Sh}_{\mathrm{x}}$ enhance. Figure 15 shows the impact of $\mathrm{Gr}_{\mathrm{b}}$ on $f^{\prime}(\eta)$. It is concluded that the present results are in good agreement with the previous studies.

\section{Conclusions}

Heat and mass transfer phenomena are inspected through radiation, joule heating, chemical reaction. The boundary layer equations are modelled and transformed to a system of
ODE'S with the aid of similarity transformations and solved numerically by utilizing R-K-F integration scheme. The graphical analysis has been emphasized for the fluid and dust phase velocity, angular velocity, energy and species fields to the influence of sundry dynamical flow quantities. In addition, friction factor, Nusselt number and Sherwood number are presented in plots and tables and discussed elaborately, and nice validation is emphasized.

1. Velocity profile diminishes for higher values of $M$

2. Thermal and species profiles enhance for rising vales of $M$

3. Compactness of momentum layer shrinkage for exaggerated values of $\mathrm{Gr}_{\mathrm{b}}, \mathrm{Gr}_{\mathrm{c}}$ and reverse behaviour is seen in angular velocity

4. Escalating values of Eckert number, radiation parameter temperature profile enhances.

5. Compactness of thermal and solutal layer decays for larger $\mathrm{Pr}, \mathrm{Sc}$ and $\mathrm{kr}$

6. Local friction factor declines for boost up values of magnetic parameter and porosity permeability parameter.

7. Rate of heat transfer decreases for increasing values of Eckert number and radiation parameter.

8. Rate of mass transfer increases for increasing values of Schmidt number and chemical reaction parameter

Open Access This article is licensed under a Creative Commons Attribution 4.0 International License, which permits use, sharing, adaptation, distribution and reproduction in any medium or format, as long as you give appropriate credit to the original author(s) and the source, provide a link to the Creative Commons licence, and indicate if changes were made. The images or other third party material in this article are included in the article's Creative Commons licence, unless indicated otherwise in a credit line to the material. If material is not included in the article's Creative Commons licence and your intended use is not permitted by statutory regulation or exceeds the permitted use, you will need to obtain permission directly from the copyright holder. To view a copy of this licence, visit http://creativecommons.org/licenses/by/4.0/.

\section{References}

1. Eringen AC. Theory of micropolar fluid. J Math Mech. 1966;16:1-18.

2. Peddison J, McNitt RP. Boundary layer theory for micropolar fluid. Recent Adv Eng Sci. 1970;5:405-26.

3. Khonsari MM, Brewe DE. Effects of viscous dissipation on the lubrication characteristics of micropolar fluids. Acta Mech. 1994;105:57-68.

4. Qukaszewicz G. Micropolar fluids theory and application. Basel: Birkhäuser; 1999.

5. Das K. Effect of chemical reaction and thermal radiation on heat and mass transfer flow of MHD micropolar fluid in a rotating frame of reference. Int J Heat Mass Transf. 2011;54:3505-13.

6. Pal D, Chatterjee S. Heat and mass transfer in MHD non-Darcian flow of a micropolar fluid over a stretching sheet embedded in a 
porous media with non-uniform heat source and thermal radiation. Commun Nonlinear Sci Nonlinear Simul. 2010;15(7):1843-57.

7. Khalid A, Khan I, Khan A, Shafie S. Influence of wall couple stress in MHD flow of a micropolar fluid in a porous medium with energy and concentration transfer. Results Phys. 2018;9:1172-84.

8. Hayat T, Sajjad R, Ellahi R, Alsaedi A, Muhammad T. Homogeneous-heterogeneous reactions in MHD flow of micropolar fluid by a curved stretching surface. J Mol Liq. 2017;240:209-20.

9. Gnaneswara Reddy M, Rama Subba Reddy G. Micropolar fluid flow over a nonlinear stretching convectively heated vertical surface in the presence of cattaneo-christov heat flux and viscous dissipation. Front Heat Mass Transf (FHMT). 2017. https://doi. org/10.5098/hmt.8.20

10. Gnaneswara Reddy M. Magnetohydrodynamics and radiation effects on unsteady convection Flow of micropolar Fluid Past a vertical vorous vlate with variable vall veat vlux. Int Sch Res Netw ISRN Thermodyn 2012; Article ID 146263.

11. Saffman G. On the stability of laminar flow of a dusty gas. J Fluid Mech. 1962;13(1962):120-8

12. Chamkha AJ. The stokes problem for a dusty fluid in the presence of magnetic field, heat generation and wall suction effects. Int $\mathrm{J}$ Numer Methods Heat Fluid Flow. 2000;10(1):116-33.

13. Mahanthesh B, Gireesha BJ. Thermal Marangoni convection in two-phase flow of dusty Casson fluid. Results Phys. 2018;8:537-44.

14. Mamatha Upadhya S, Raju CSK, Saleem S, Alderremy AA. Modified Fourier heat flux on MHD flow over stretched cylinder filled with dust, Graphene and silver nanoparticles. Results in Phys. 2018;9:1377-85

15. Ghadikolaei SS, Hosseinzadeh Kh, Ganji DD. MHD raviative boundary layer analysis of micropolar dusty fluid with graphene oxide (Go)- engine oil nanoparticles in a porous medium over a stretching sheet with joule heating effect. Powder Technol. 2018;338:425-37.

16. Gnaneswara Reddy M, Sudha Rani MVVNL, Ganesh Kumar K, Prasannakumara BC. Cattaneo-Christov heat flux and non-uniform heat-source/sink impacts on radiative Oldroyd-B two-phase flow across a cone/wedge. J Braz Soc Mech Sci Eng. 2018;40:95. https://doi.org/10.1007/s40430-018-10338.

17. Gnaneswara Reddy M, Sudha Rani MVVNL, Prasannakumara C. Non-linear radiation and chemical reaction impacts on hydromagnetic particle suspension flow in non-Newtonian fluids. Int $\mathrm{J}$ Chem Reactor Eng. 2018;16:20180001.

18. Souayeh B, Kumar KG, Reddy MG, Rani S, Hdhiri N, Alfannakh $\mathrm{H}$, Rahimi-Gorji M. Slip flow and radiative heat transfer behavior of Titanium alloy and ferromagnetic nanoparticles along with suspension of dusty fluid. J Mol Liq. 2019;290:111223.

19. Qayyum S, Hayat T, Shehzad SA, Alsaedi A. Mixed convection and heat generation/absorption aspects in MHD flow of tangenthyperbolic nanoliquid with Newtonian heat/mass transfer. Radiat Phys Chem. 2018;144:396-404.

20. Vo DD, Hedayat M, Ambreen T, Shehzad SA, Sheikholeslami M, Shafe A, Nguyen TK. Effectiveness of various shapes of $\mathrm{Al}_{2} \mathrm{O}_{3}$ nanoparticles on the MHD convective heat transportation in porous medium: CVFEM modeling. J Therm Anal Calorim. 2020;139:1345-53.

21. Alamri Sultan Z, Ellahi R, Shehzad N, Zeeshan A. Convective radiative plane Poiseuille flow of nanofluid through porous medium with slip: an application of Stefan blowing. J Mol Liq. 2019;273:292-304.

22. Bhatti MM, Zeeshan A, Ellahi R. Heat transfer with thermal radiation on MHD particle-fluid suspension induced by metachronal wave. Pramana J Phys. 2017;89(3):48.

23. Bhatti MM, Zeeshan A, Ellahi R, AnwarBég O, Kadir A. Effects of coagulation on the two-phase peristaltic pumping of magnetized Prandtl biofluid through an endoscopic annular geometry containing a porous medium. Chin J Phys. 2019;58:222-34.

24. Ellahi R, Sait SM, Shehzad N, Mobin N. Numerical simulation and mathematical modeling of electro-osmotic Couette-Poiseuille flow of MHD power-law nanofluid with entropy generation. Symmetry. 2019;11(8):1038.

25. Khan LA, Raza M, Mir NA, Ellahi R. Effects of different shapes of nanoparticles on peristaltic flow of MHD nanofluids filled in an asymmetric channel: a novel mode for heat transfer enhancement. J Therm Anal Calorim. 2019. https://doi.org/10.1007/s1097 3-019-08348-9.

26. Sheikholeslami M, Gerdroodbary MB, Moradi R, Shafee A, Li $\mathrm{Z}$. Application of neural network for estimation of heat transfer treatment of $\mathrm{Al}_{2} \mathrm{O}_{3}-\mathrm{H}_{2} \mathrm{O}$ nanofluid through a channel. Comput Methods Appl Mech Eng. 2019;344:1-12.

27. Raei B, Shahraki F, Jamialahmadi M, Peyghambarzadeh SM. Experimental investigation on the heat transfer performance and pressure drop characteristics of $\gamma-\mathrm{Al}_{2} \mathrm{O}_{3}$ /water nanofluid in a double tube counter flow heat exchanger. J Therm Anal Calorim. 2017; 127:2561-75.

28. Monge-Palacios $\mathrm{M}$, Rafatijo $\mathrm{H}$. On the role of the termolecular reactions $2 \mathrm{O}_{2}+\mathrm{H}_{2} \rightarrow 2 \mathrm{HO}_{2}$ and $2 \mathrm{O}_{2}+\mathrm{H}_{2} \rightarrow \mathrm{H}+\mathrm{HO}_{2}+\mathrm{O}_{2}$ in formation of the first radicals in hydrogen combustion: ab initio predictions of energy barriers. J Therm Anal Calorim. 2018;133:289-301.

29. Sheikholeslami M, Rokni HB. Melting heat transfer influence on nanofluid flow inside a cavity in existence of magnetic field. Int J Heat Mass Transf. 2017;114:517-26.

30. Sheikholeslami M, Shafee A, Zareei A, Haq R-U, Li Z. Heat transfer of magnetic nanoparticles through porous media including exergy analysis. J Mol Liq. 2019;279:719-32.

31. Rafatijo H, Monge-Palacios M, Thompson DL. Identifying collisions of various molecularities in molecular dynamics simulations. J Phys Chem. 2019;123:1131-9.

32. Sheikholeslami M, Vajravelu K. Nanofluid flow and heat transfer in a cavity with variable magnetic field. Appl Math Comput. 2017;298:272-82.

33. Reddy MG. Cattaneo-Christov heat flux effect on hydromagnetic radiative Oldroyd-B liquid flow across a cone/wedge in the presence of cross-diffusion. Eur Phys J Plus. 2018;133:24. https://doi. org/10.1140/epjp/i2018-11844-0.

34. Sawchuk SP, Zamir M. Boundary layer on a circular cylinder in axial flow. Int J Heat Fluid Flow. 1992;13(2):184-8.

35. Prasannakumara BC, Reddy MG, Rani MS, Krishnamurthy MR. Effect of chemical reaction on maxwell nanofluid slip flow over a stretching sheet. Int J Chem Reactor Eng. 2018;17:20180065.

36. Kalyani K, Rao NS, Makinde OD, Reddy MG, Rani MS. Influence of viscous dissipation and double stratification on MHD OldroydB fluid over a stretching sheet with uniform heat source. SN Appl Sci. 2019. https://doi.org/10.1007/s42452-019-0339-1.

37. Mather DJ. The motion of viscous liquid past a paraboloid. Quart J Mech Appl Math. 1961;14(4):423-30. https://doi.org/10.1093/ qjmam/14.4.423.

38. Lee LL. Boundary layer over a thin needle. Phys Fluids. 1967;10(1063/1):1762194.

39. Animasaun IL, Pop I. Numerical exploration of a non-Newtonian Carreau fluid flow driven by catalytic surface reactions on an upper horizontal surface of a paraboloid of revolution, buoyancy and stretching at the free stream. Alex Eng J. 2017;56:647-58.

40. Kumaran G, Sandeep N, Animasaun IL. Computational modeling of magnetohydrodynamic non-Newtonian fluid flow past a paraboloid of revolution. Alex Eng J. 2017;57:1859-65.

41. Khan M, Hussain A, Malik MY, Salahuddin T. Biconvection flow of Carreau fluid over an upper paraboloid surface: a computational study. Results Phys. 2017;7:4050-7. 
42. Animasaun IL, Sandeep N. Buoyancy induced model for the flow of $36 \mathrm{~nm}$ alumina-water nanofluid along upper horizontal surface of paraboloid of revolution with variable thermal conductivity and viscosity. Powder Technol. 2016;301:858-67.
Publisher's Note Springer Nature remains neutral with regard to jurisdictional claims in published maps and institutional affiliations. 